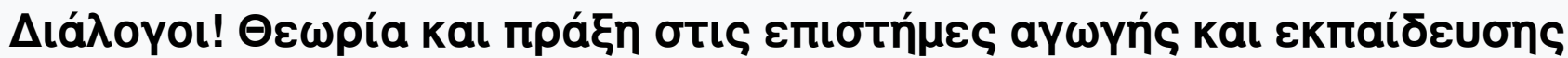

Tóp. 1 (2015)

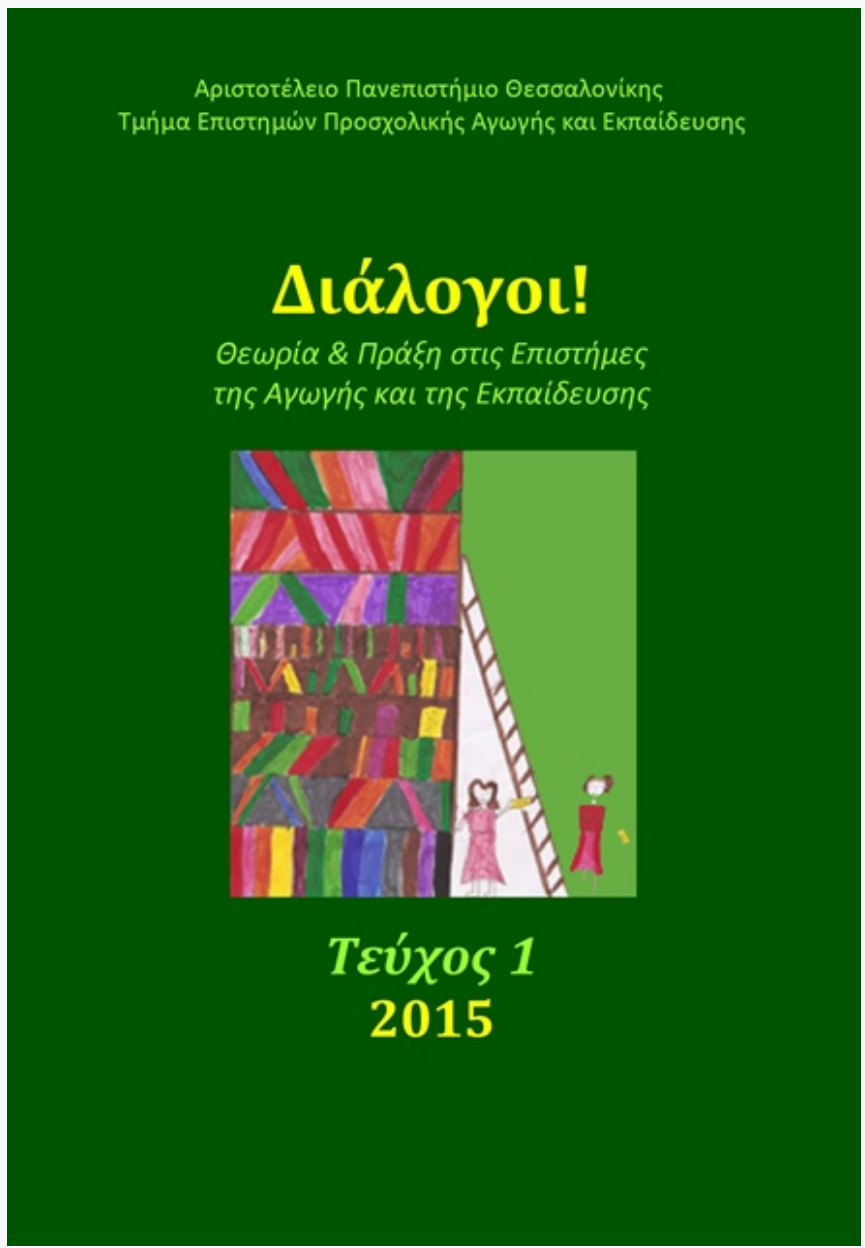

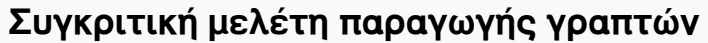

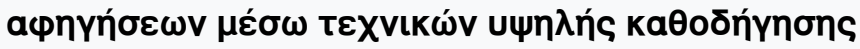

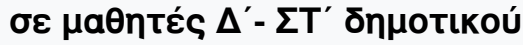

\section{Styliani Xanthi}

doi: $\underline{10.12681 / \text { dial.1983 }}$

\section{Copyright @ 2016, Styliani Xanthi}

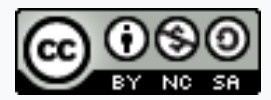

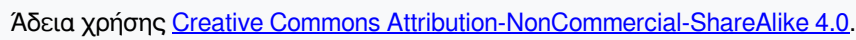

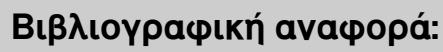

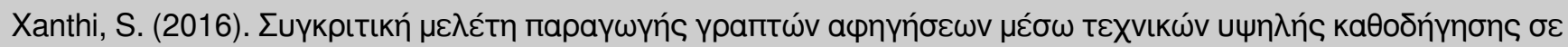

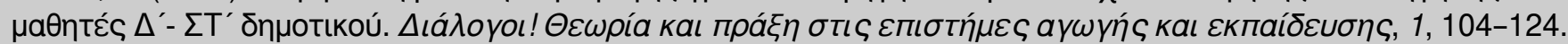
https://doi.org/10.12681/dial.1983 


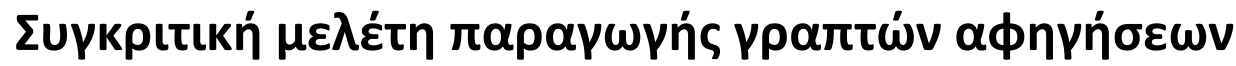

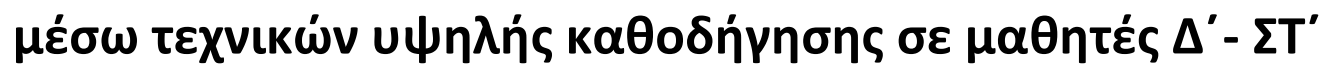 ठпнотккоن́
}

\author{
$\Sigma \tau u \lambda\llcorner a v \eta ́ ~ \Xi \alpha ́ v \theta \eta$

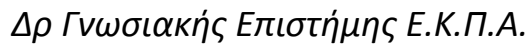

\begin{abstract}
Пвріं $\lambda \eta \Psi \eta$

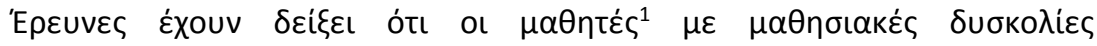

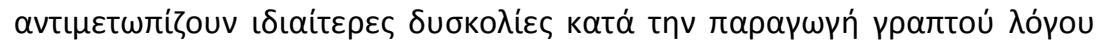

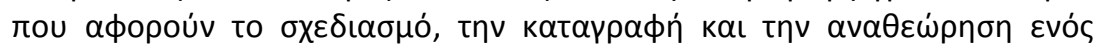

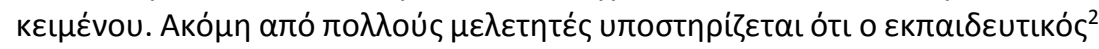

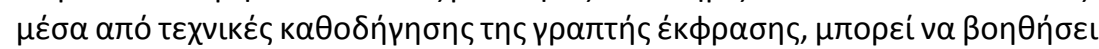

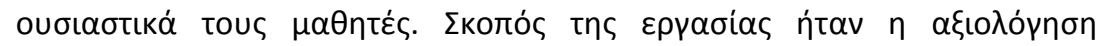

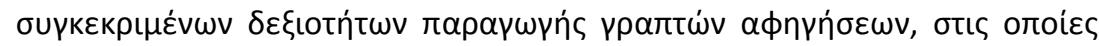

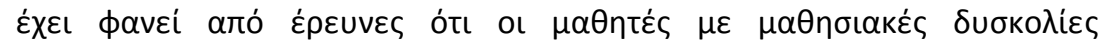

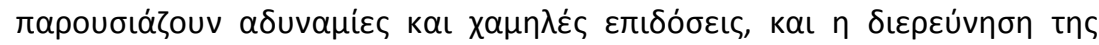

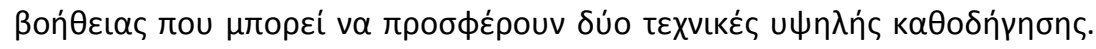

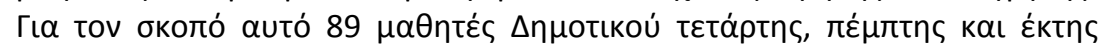

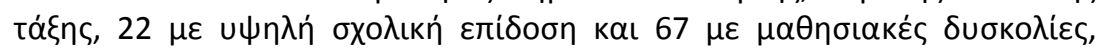

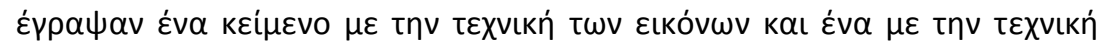

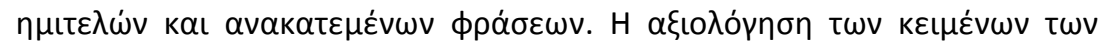

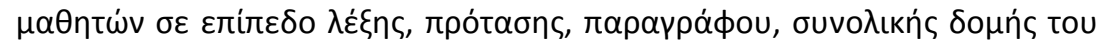

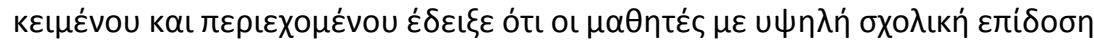

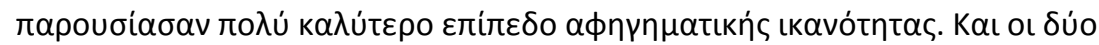

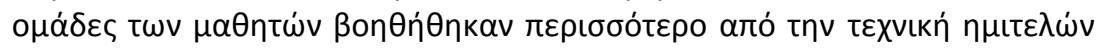

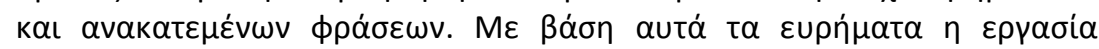

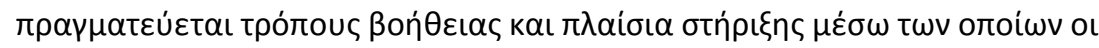

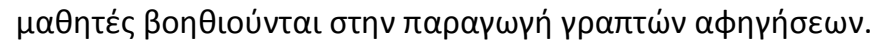

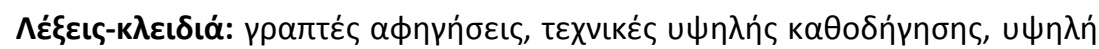

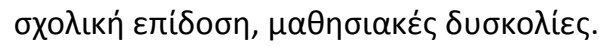

\begin{abstract}
Studies have shown that the pupils with learning disabilities experiencing particular difficulties in the writing skills related to the planning, the recording and the revising of the text. Their texts are short usually, with incomplete content and with problems in the spelling and the punctuation. There is a clear lack in their organization, the vocabulary is limited and their sentences have poor structure. They also present inability to give in writing their ideas about a topic and they use non-functional elements in their text. They write with a simplistic way just quoting one information after another. So the information seems unconnected and the text is inconsistent. In addition they have limited metacognitive skills. Regarding the role of the teacher multiple
\end{abstract}

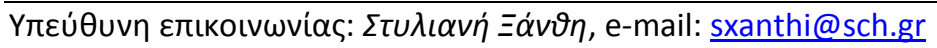


studies indicate his mediator, facilitator and supportive role, as the pupilwriter writes his own texts. Specifically many scholars argue that the teacher can help the pupils through the technical guidance of written expression. For the support frameworks of thoughts and the expression of ideas of pupils there are numerous techniques, high or loose guidance, from which can be chosen depending on the genre and the level of pupils. In the Greek Elementary textbooks of Language are used heavily two technical of high guidance, the technique of images and the technique of unfinished and muddled phrases for narrative texts. The aim of this study was to assess specific production skills of written narratives, which has been shown by research that the pupils with learning disabilities show weak and poor performance. In addition another aim was to investigate the assistance that offer two technical of high guidance, the technique of images and the technique of unfinished and muddled phrases, to pupils 9 to 12 years old. We thought that such investigation will give us the opportunity for useful conclusions for effective teaching to pupils who have particular difficulties in the production of narrative texts. For this purpose 89 pupils of the last three grades of primary school, 22 with high school performance and 67 with learning disabilities, wrote two narrative texts, one with the technique of images and one with the technique of unfinished and muddled phrases. It was assumed that the pupils with learning disabilities will be expressed with poor vocabulary and they will show lower level of narrative capacity not using the appropriate language and grammatical structures. Their texts will be short with incomplete content and with problems in the spelling and the punctuation. Both techniques will also help pupils to produce quality texts with their help. The pupils' writing skills was evaluated by comparing their written texts with two techniques, in the word level, in sentence level, in paragraph level, in overall structure of the text and in the content. The results show that the technique of unfinished and muddled phrases helped more both groups of pupils to write more extensive texts and to express with better linguistic style. As expected the pupils with high school performance showed much better level of narrative capacity using appropriate language and grammatical structures. They were expressed with richer vocabulary and they acknowledged better structural elements of narrative discourse. Their texts included more references about the environment, the goals and the emotions of the protagonists. Based on these findings, the present study discusses how the teacher can guide better the thoughts of pupils to write narrative texts and help them to express their ideas effectively. Additional it discusses the role of a supporting framework that functions as mental collaborator of pupil and which involves him actively in the structuring of knowledge strengthens his critical thought and develops cognitive and metacognitive skills.

Keywords: written narratives, high technical guidance, high school performance, learning disabilities.

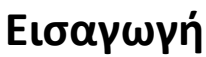

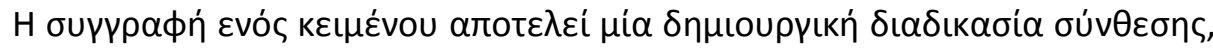

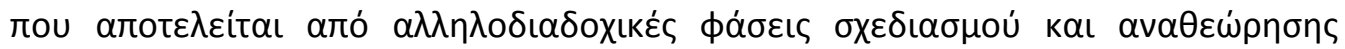

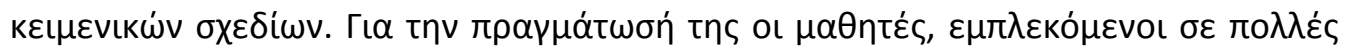

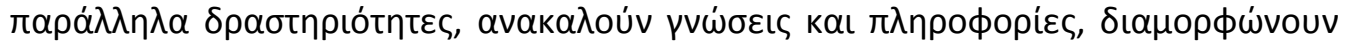




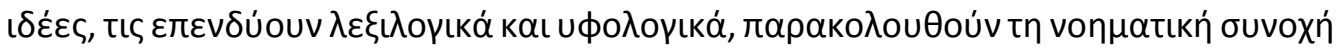

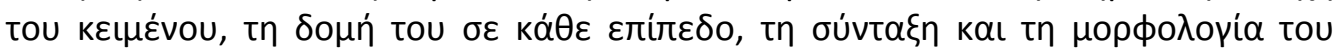

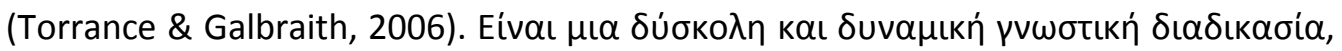

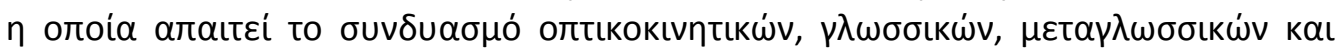

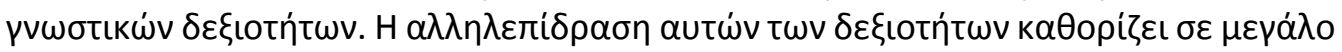

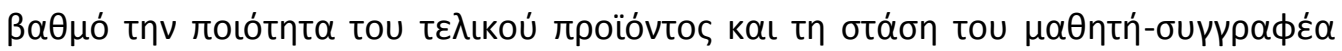

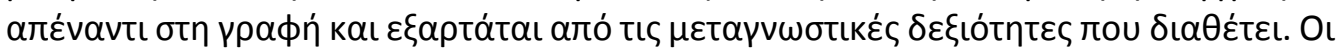

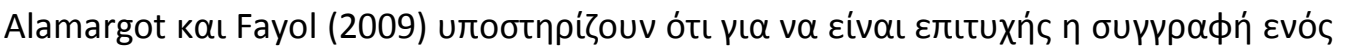

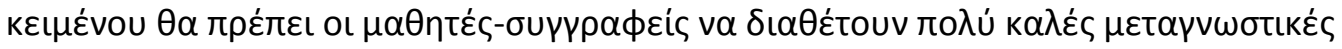

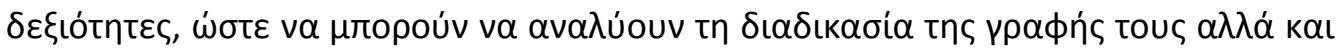

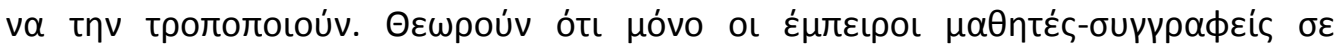

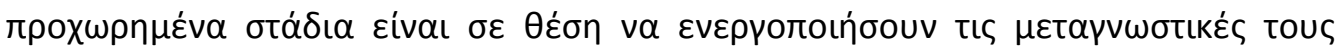

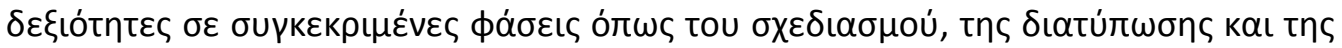

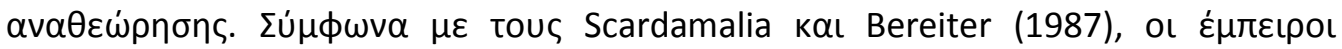

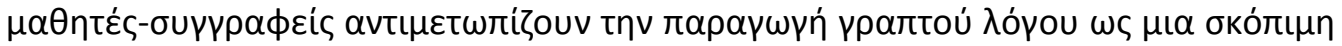

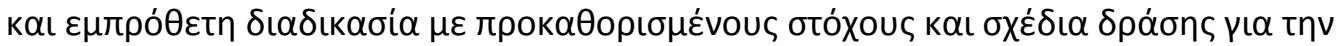

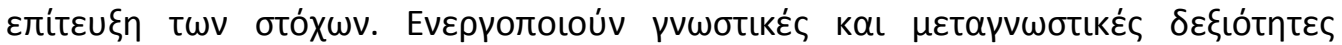

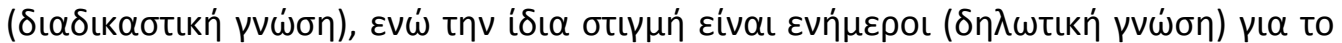

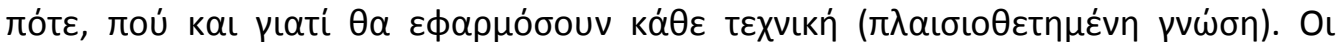

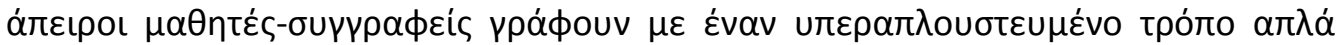

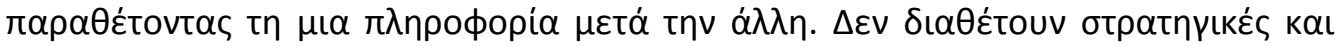

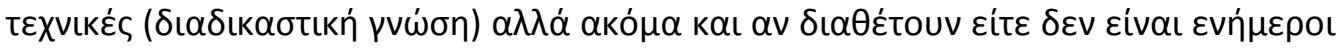

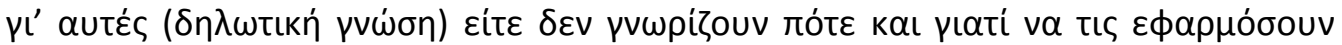

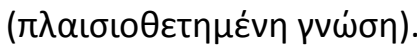

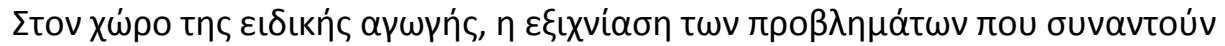

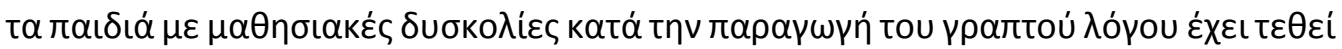

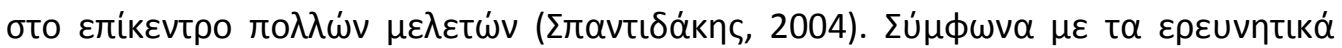

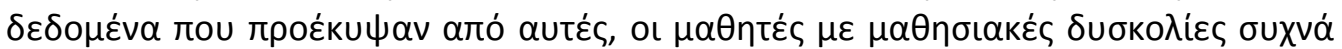

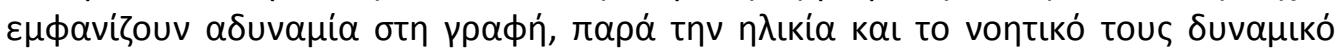

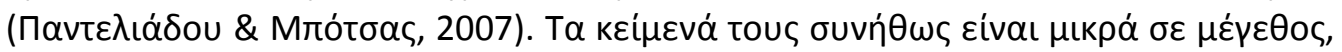

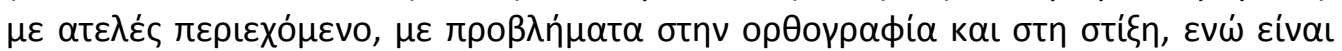

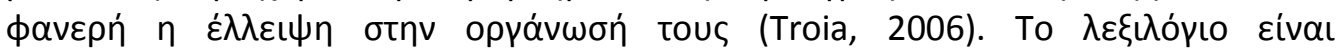

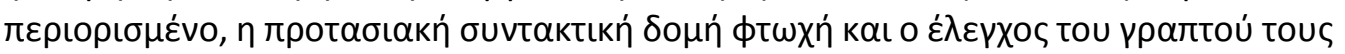

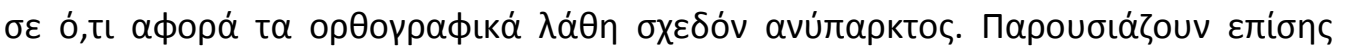

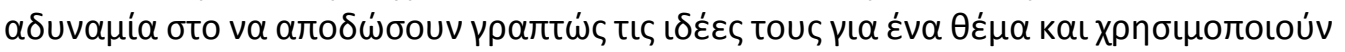

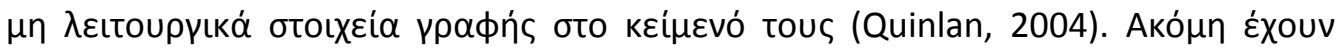

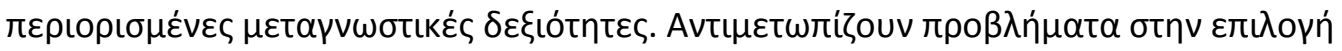

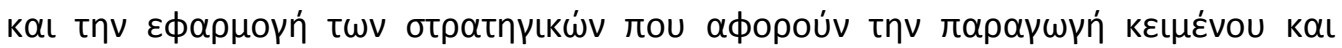

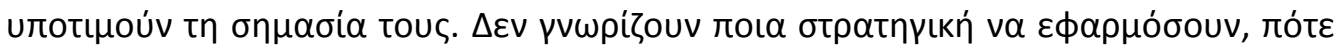

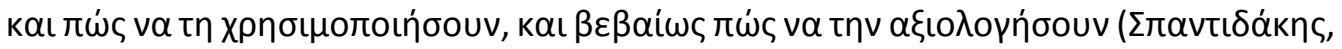

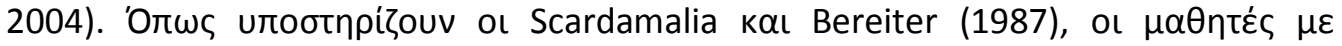

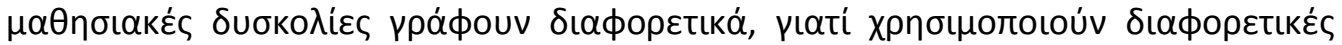

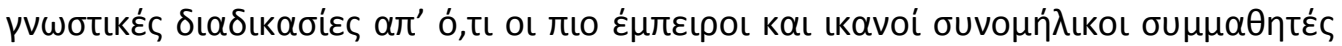

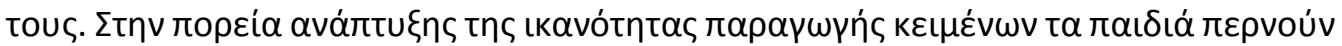

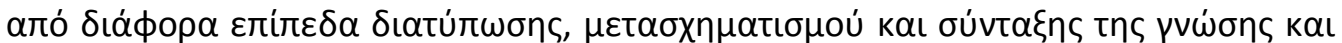

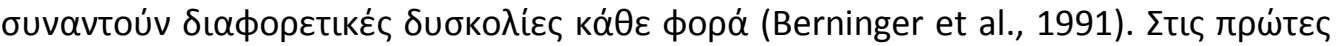

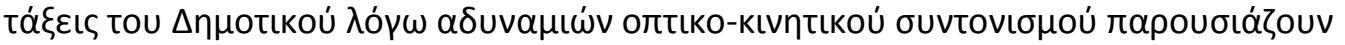




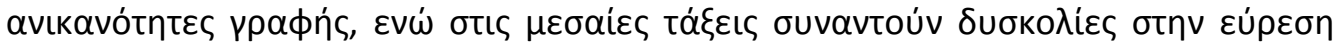

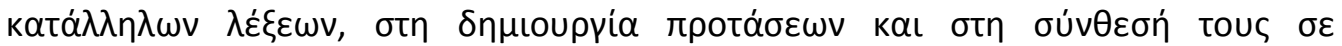

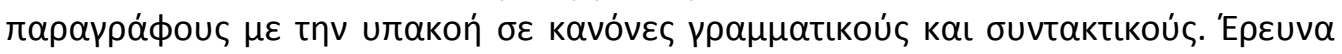

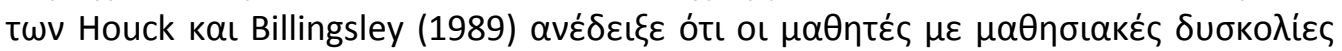

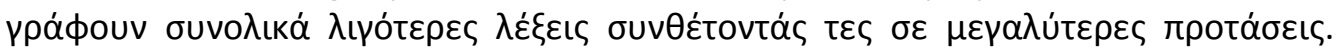

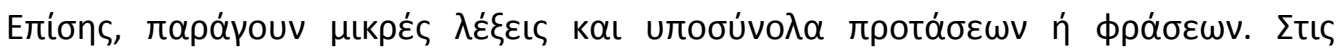

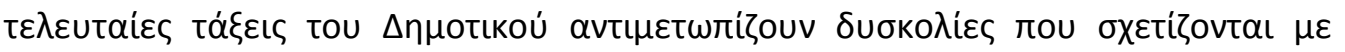

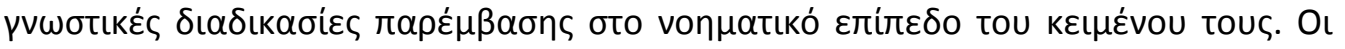

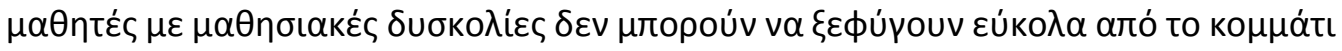

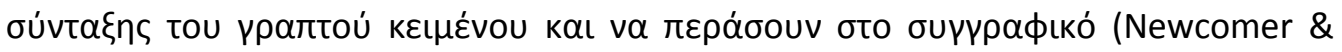

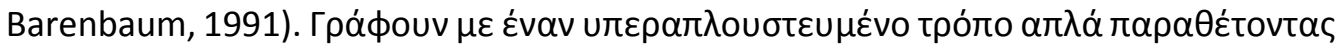

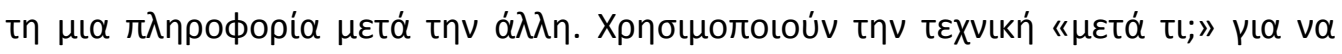

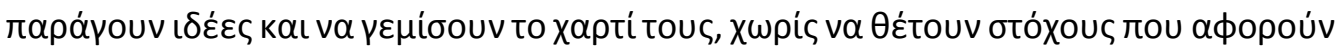

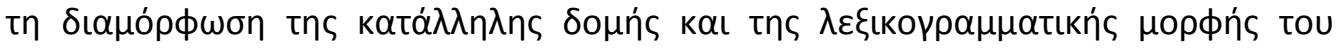

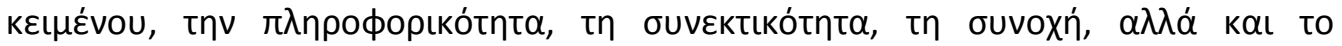

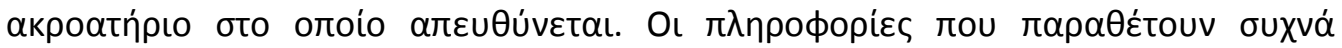

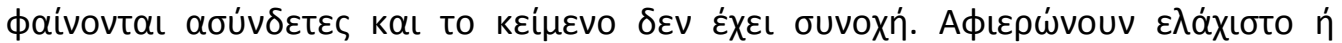

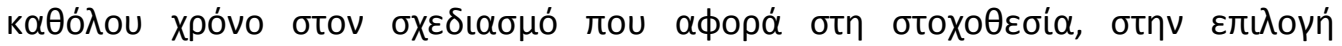

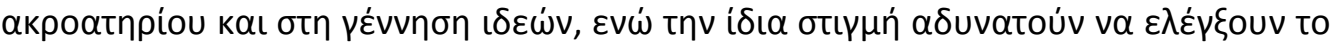

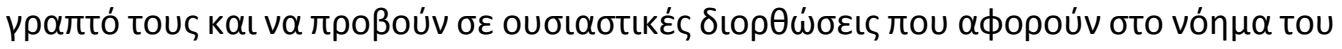

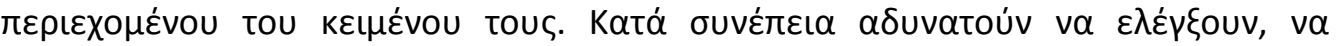

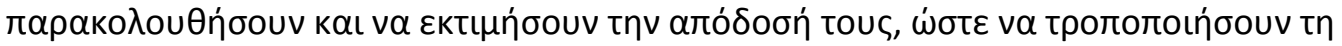

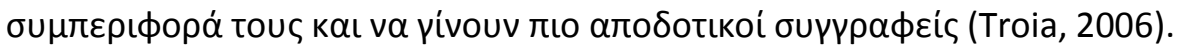

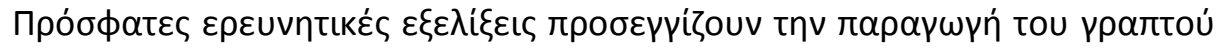

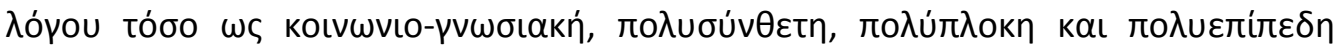

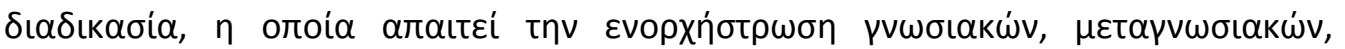

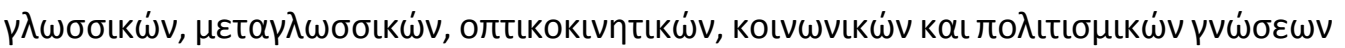

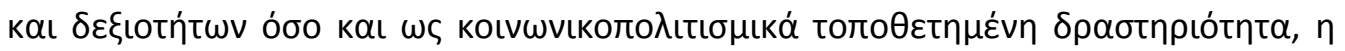

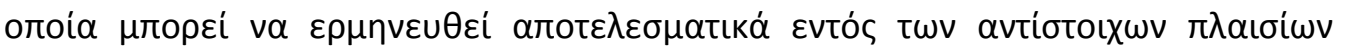

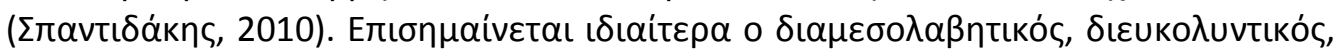

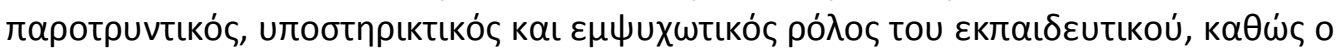

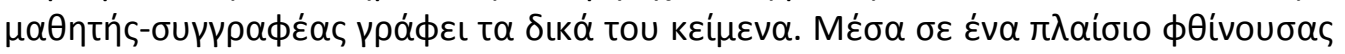

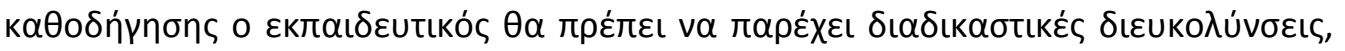

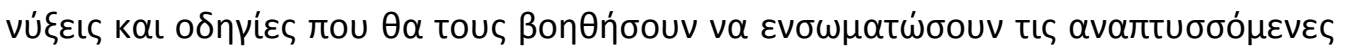

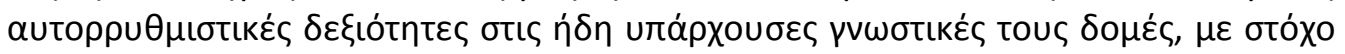

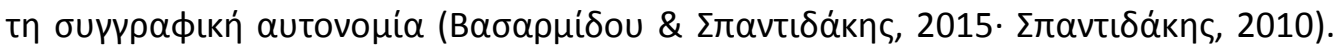
Y

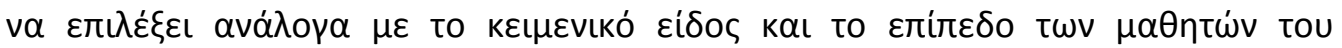

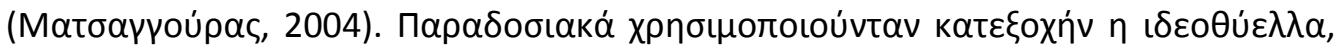

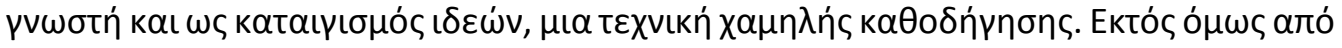

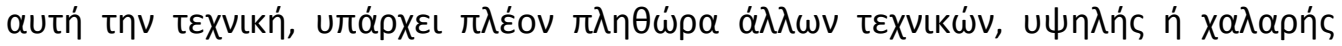

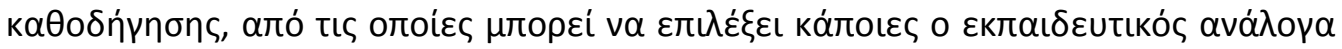

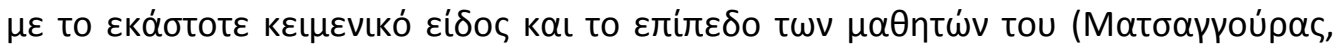

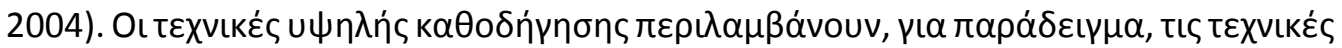

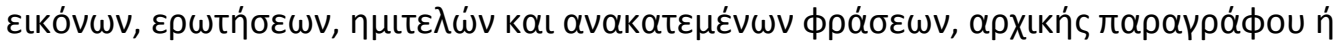

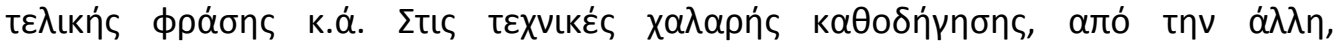

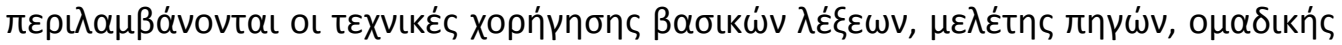




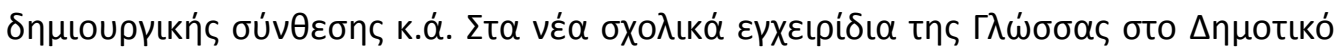

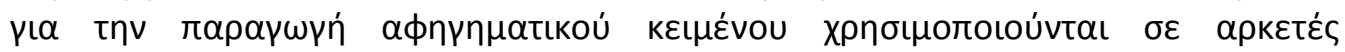

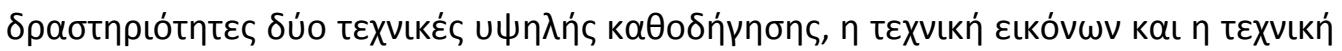

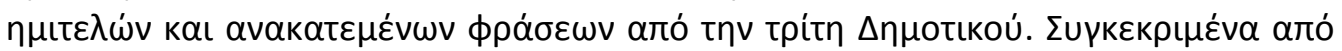

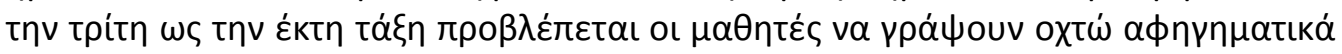

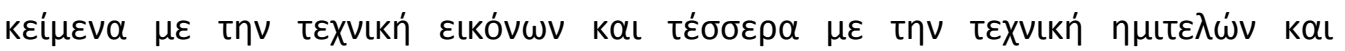

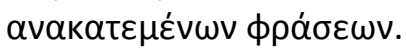

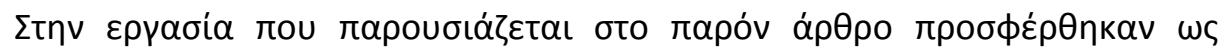

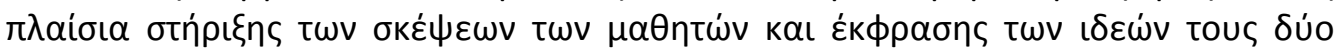

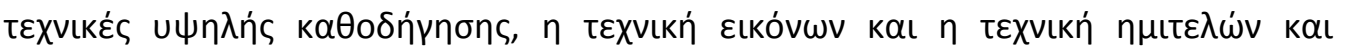

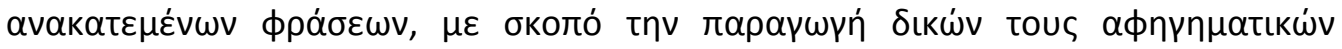

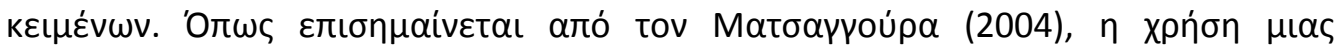

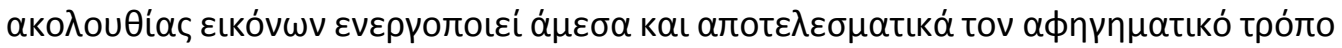

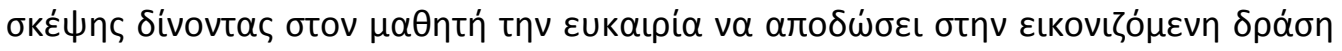

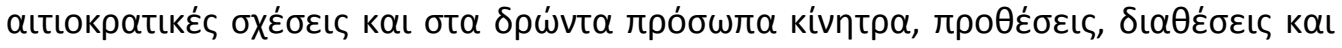

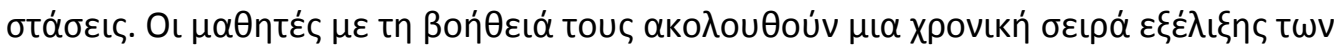

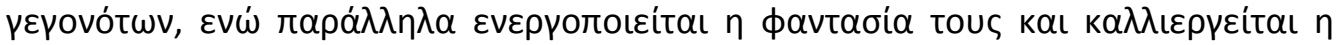

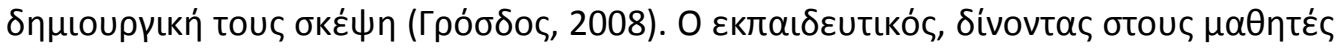

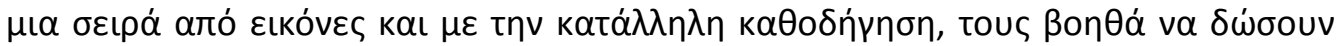

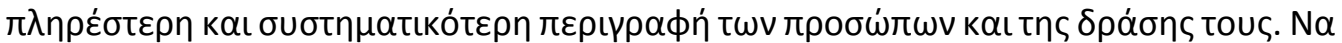

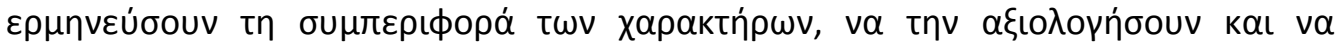

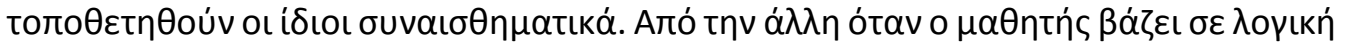

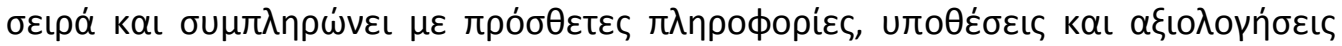

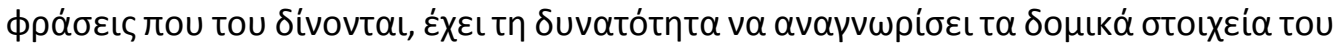

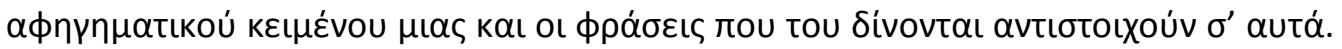

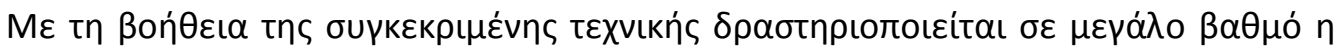

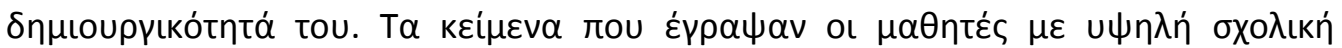

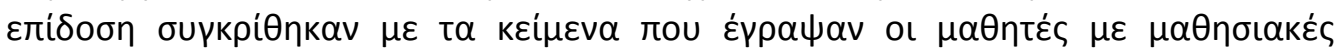

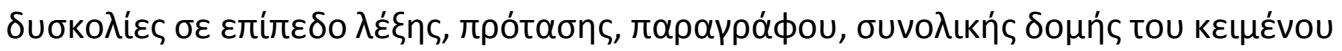

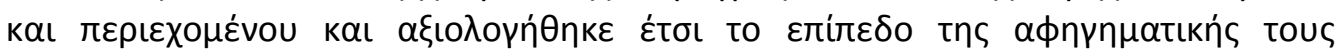

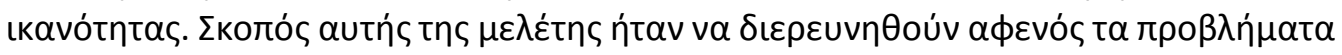

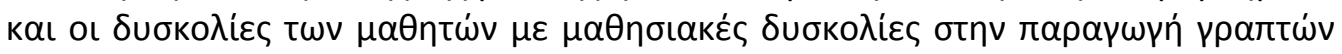

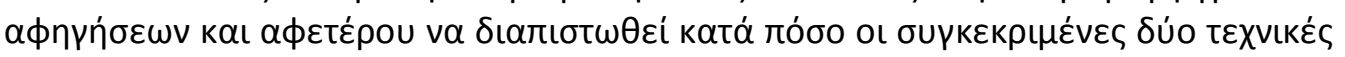

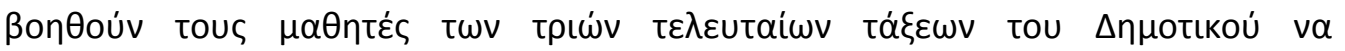

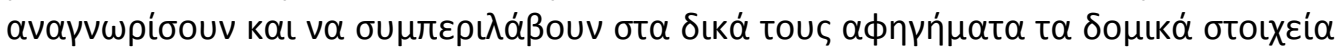

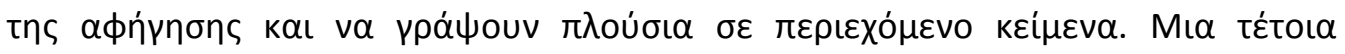

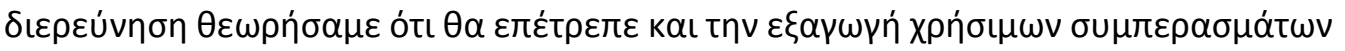

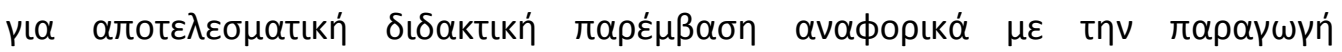
$\alpha \phi \eta \eta \eta \mu \alpha \tau$ เ

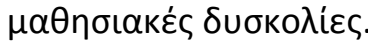

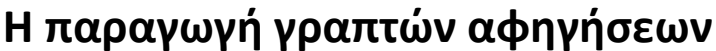

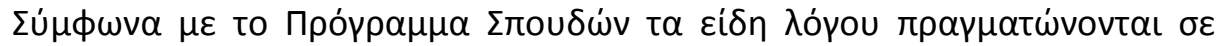

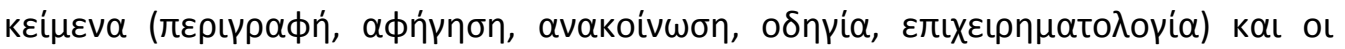

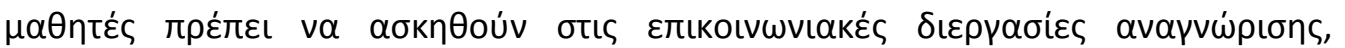

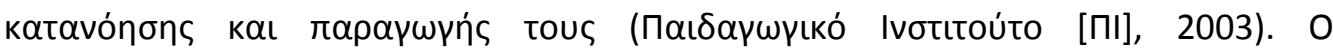




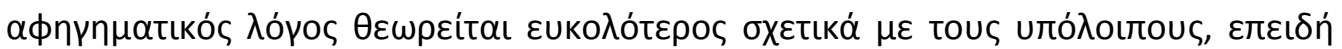

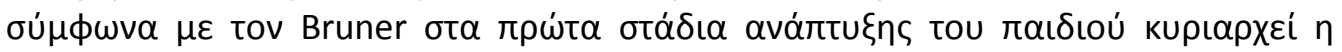
$\alpha \phi \eta \eta \eta \mu \alpha \tau$ เ

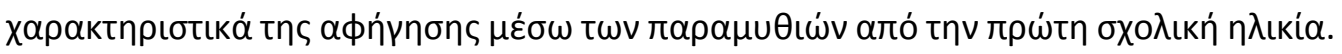

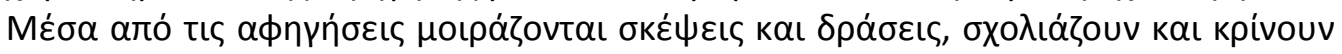

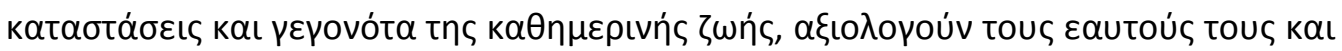

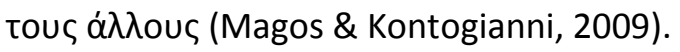

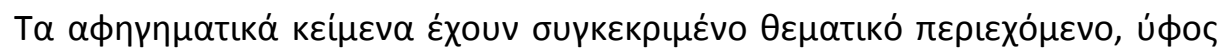

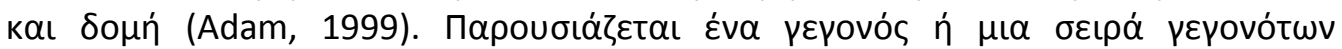

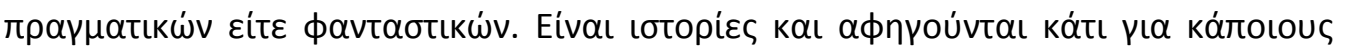

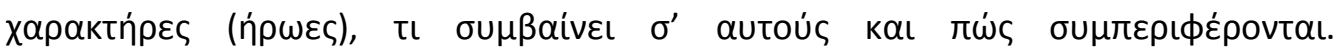

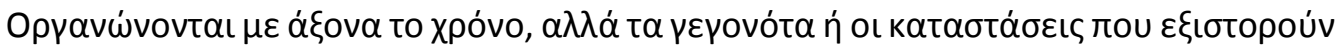

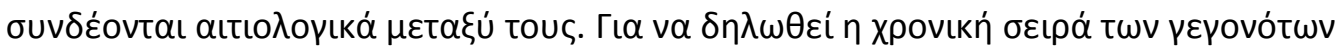

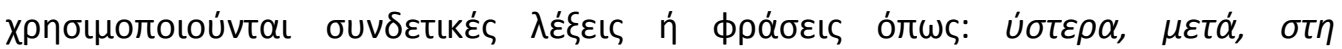

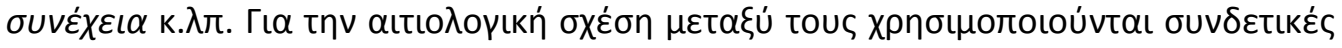

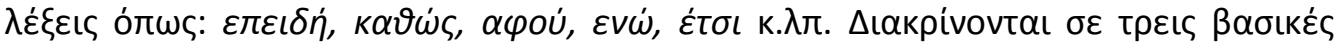

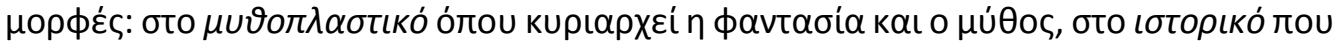

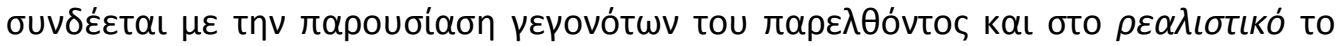

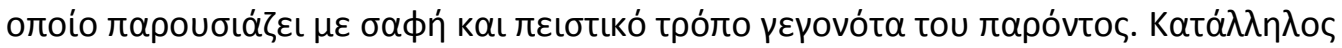

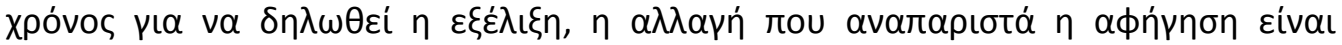

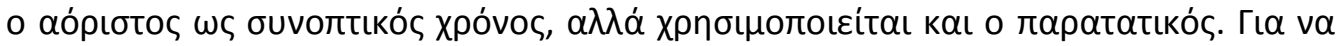

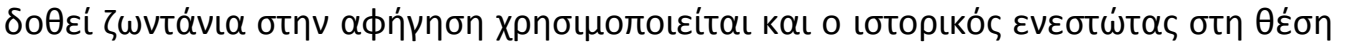

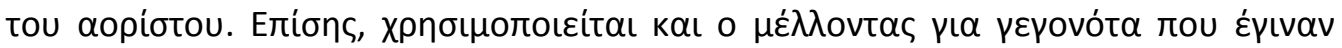

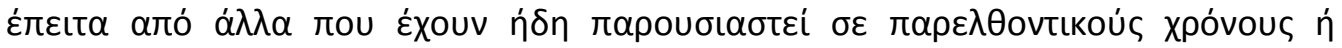

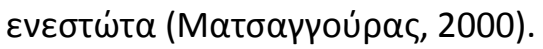

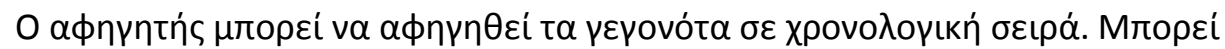

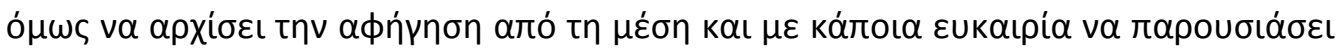

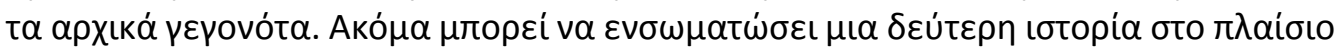

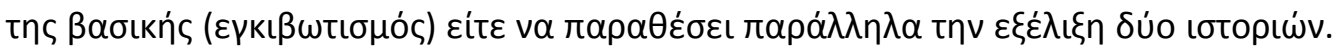

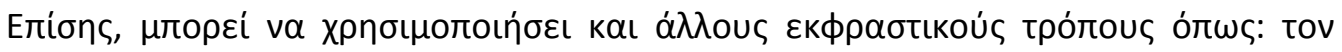

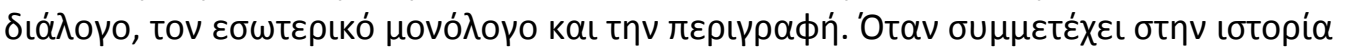

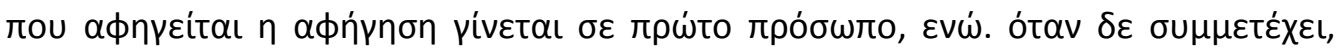

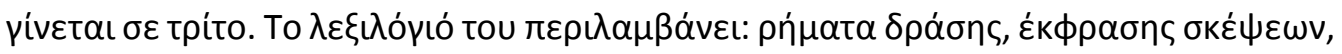

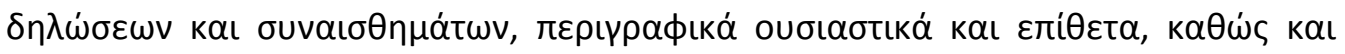

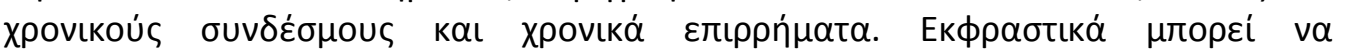

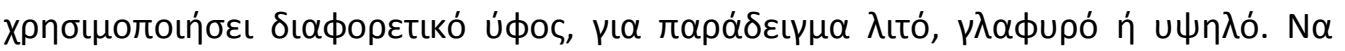

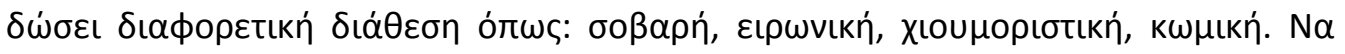

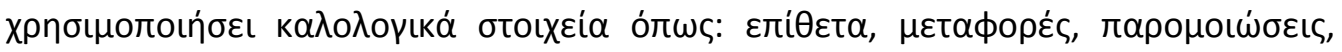

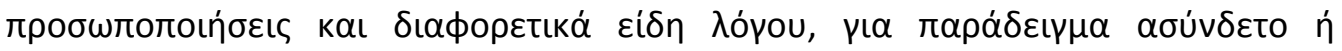

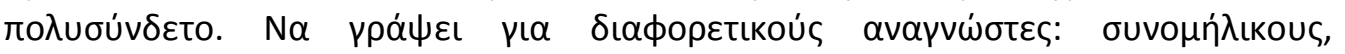

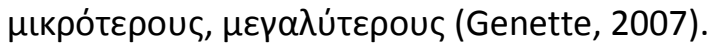

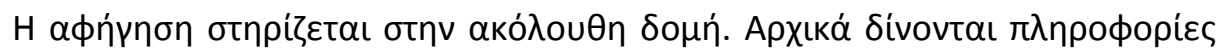

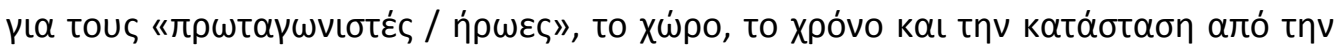

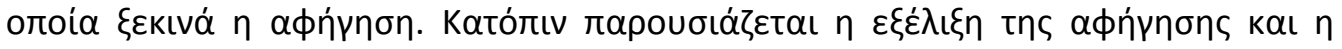

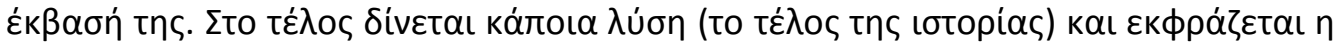

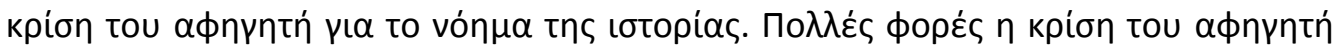




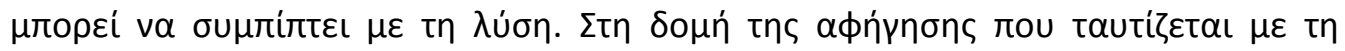

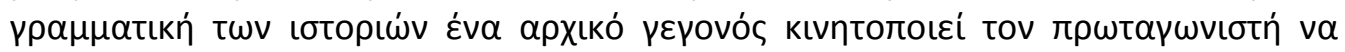

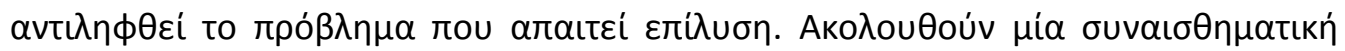

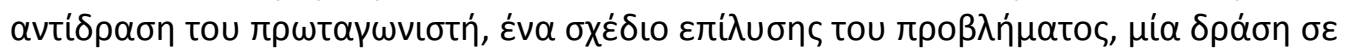

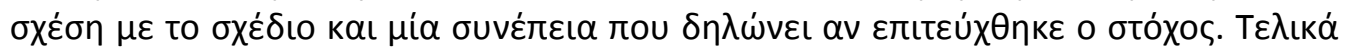

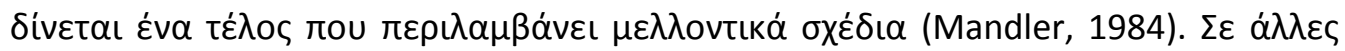

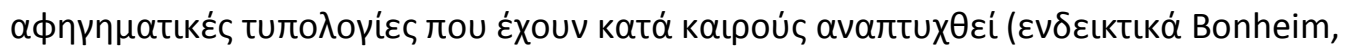

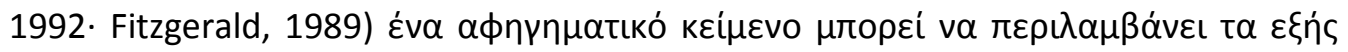

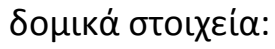

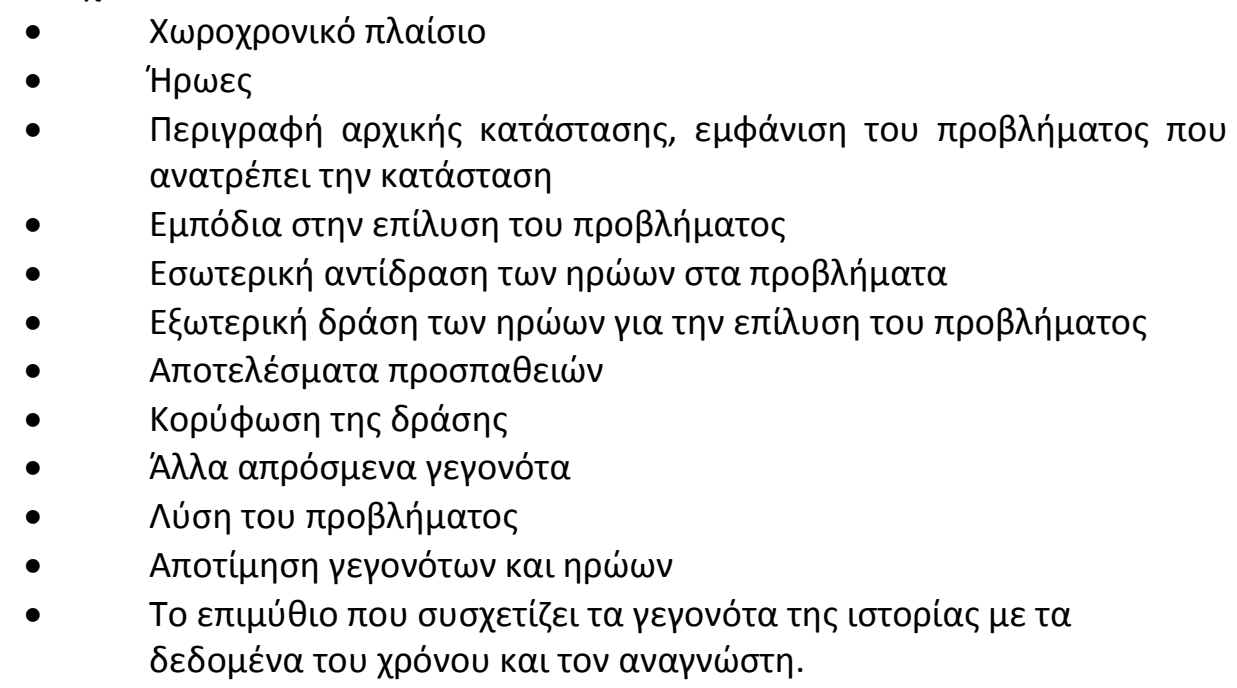

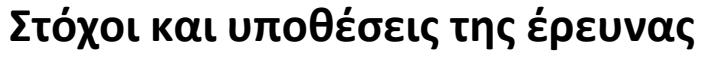

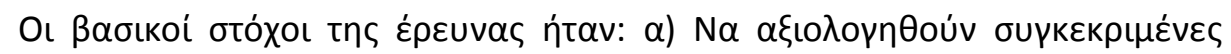

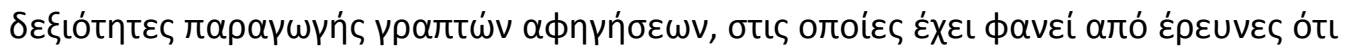

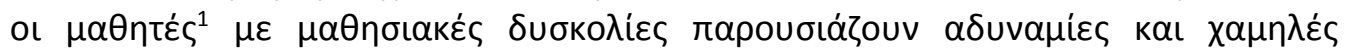

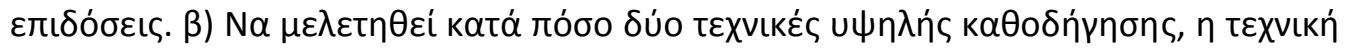

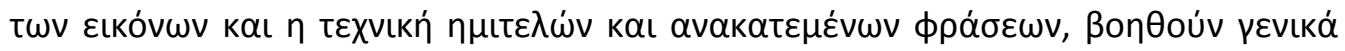

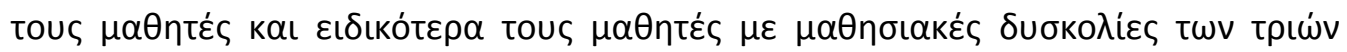

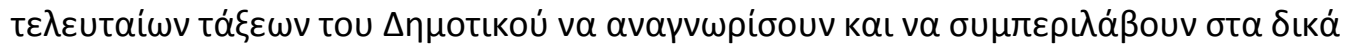

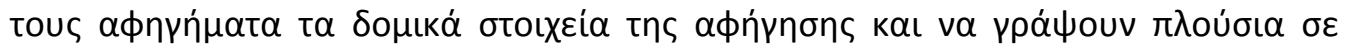

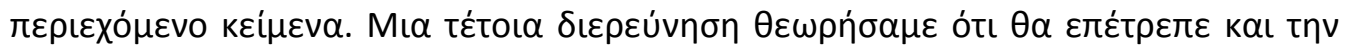

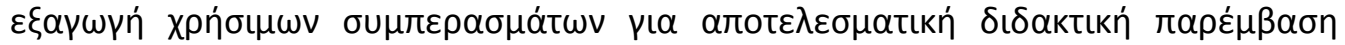

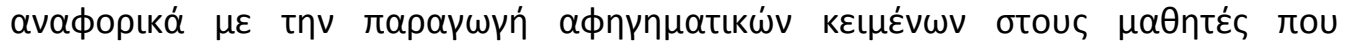

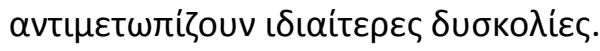

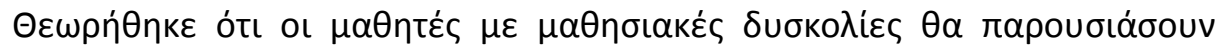

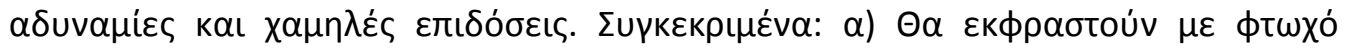

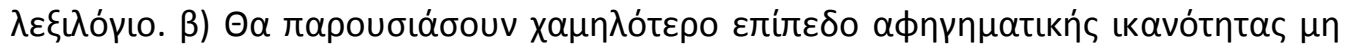

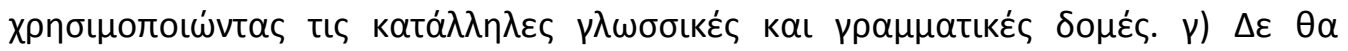

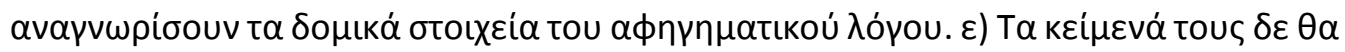

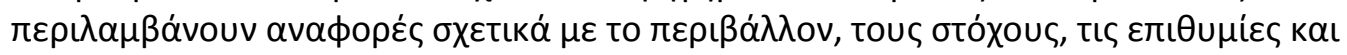

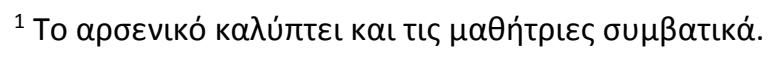




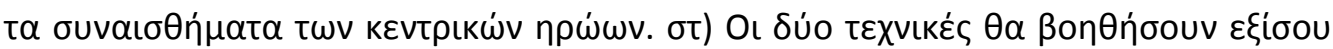

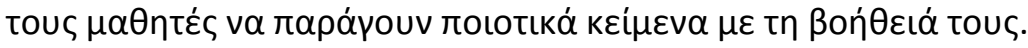

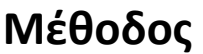

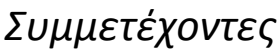

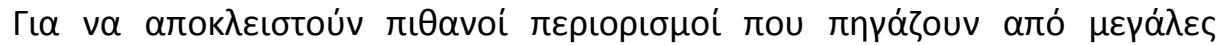

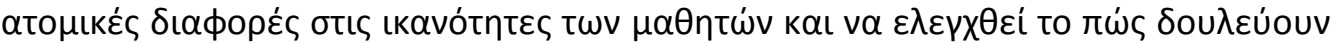

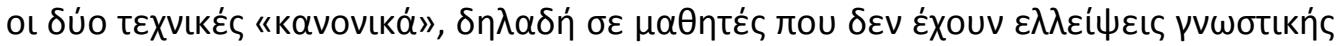

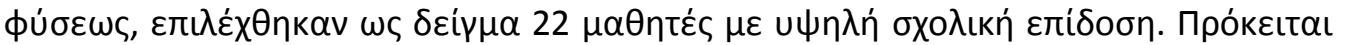

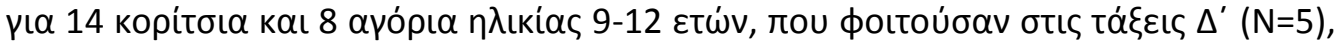

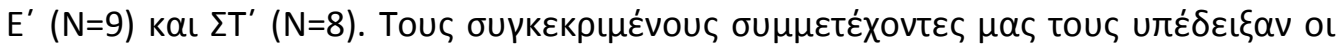

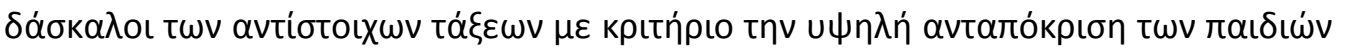

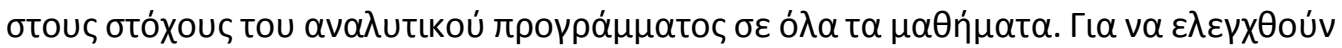

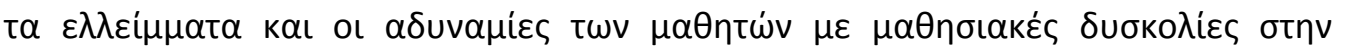

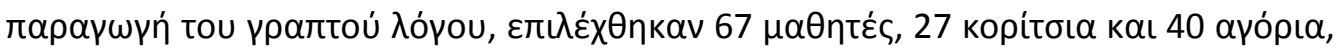

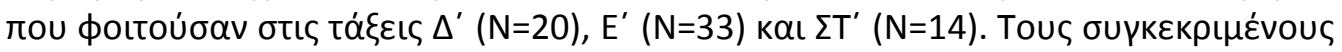

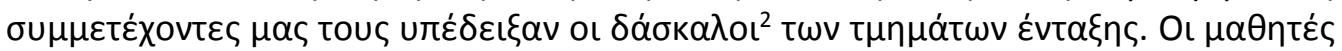

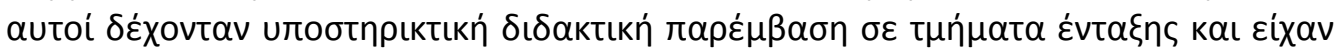

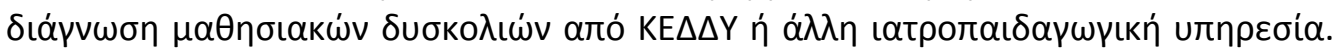

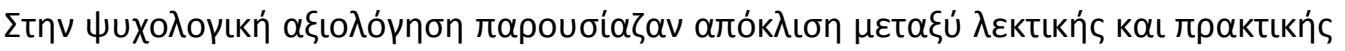

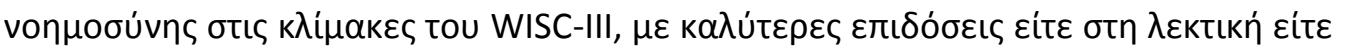

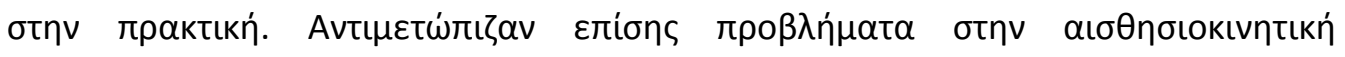

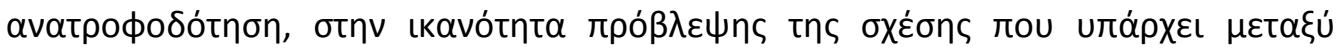

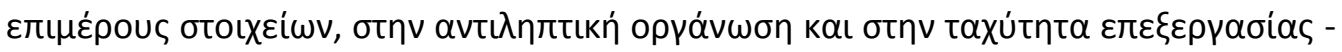

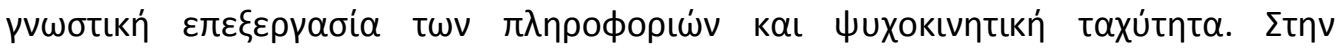

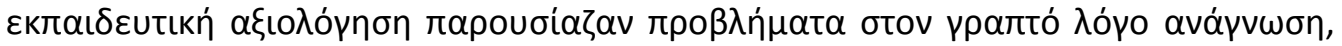

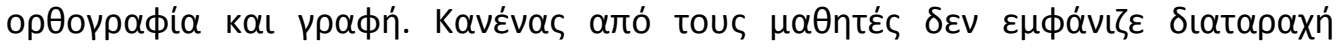

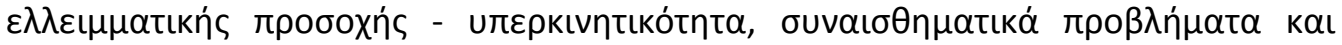

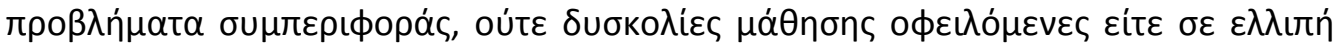

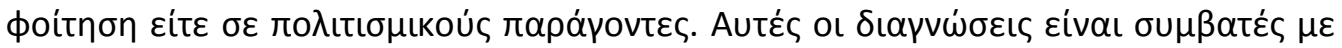

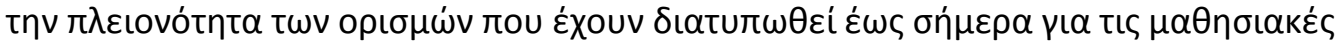

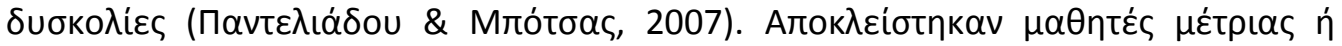

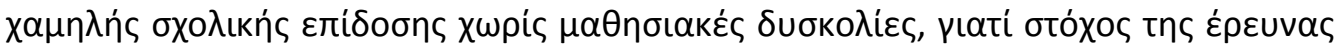

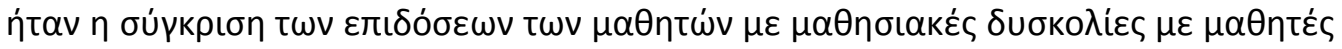

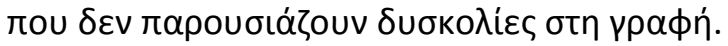

\section{$\Delta \iota \alpha \delta \iota \alpha \sigma i \alpha$}

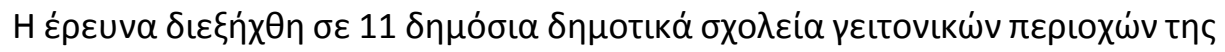

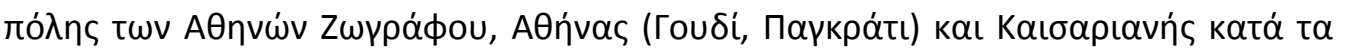

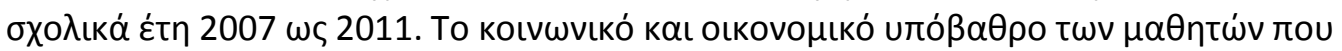

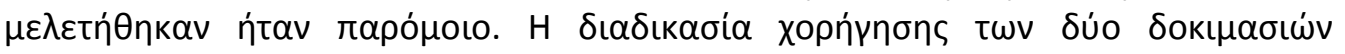

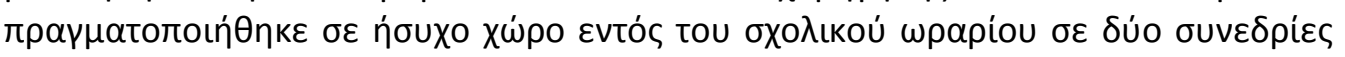

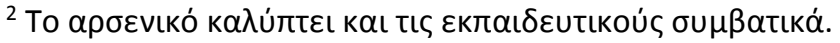




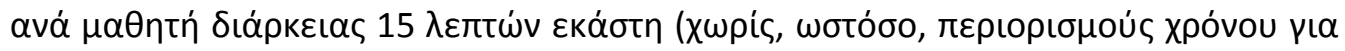

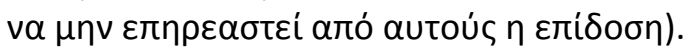

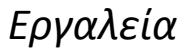

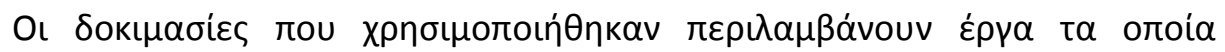

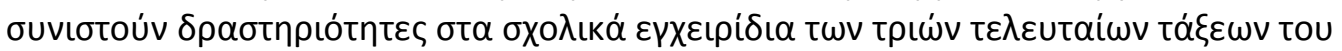

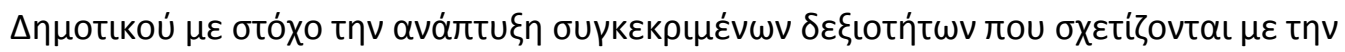

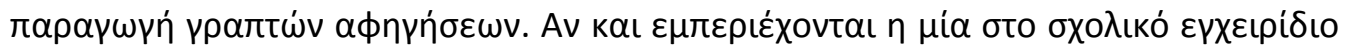

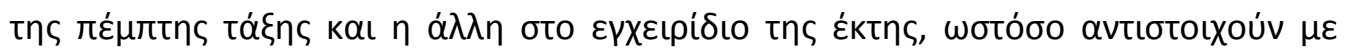

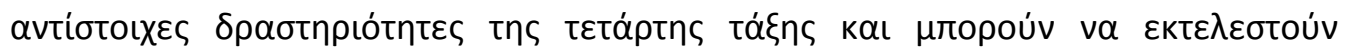

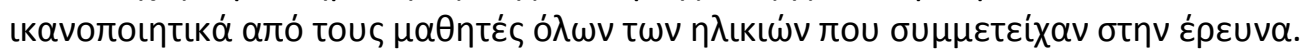

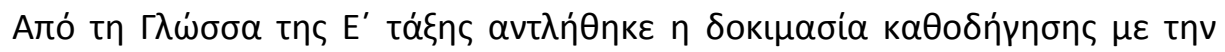

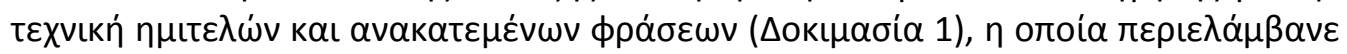

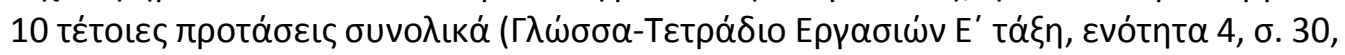

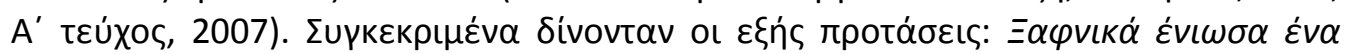

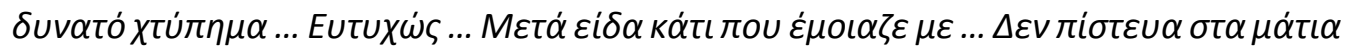

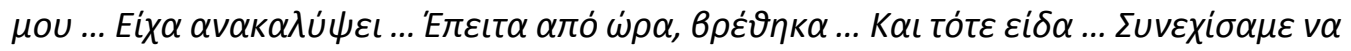

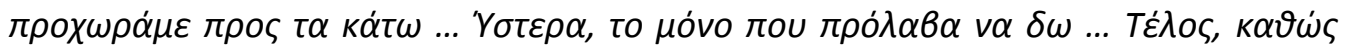

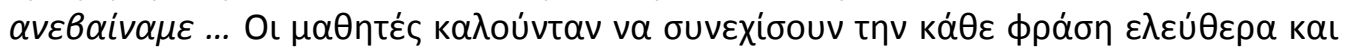

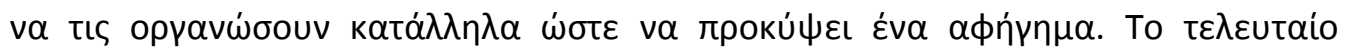

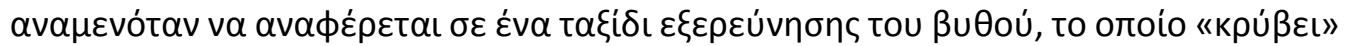

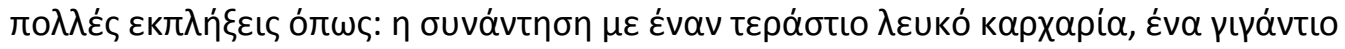

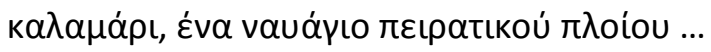

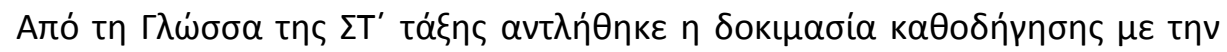

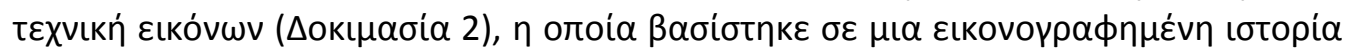

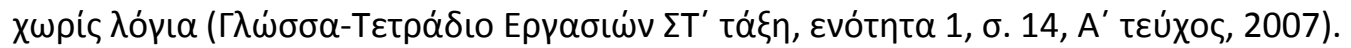

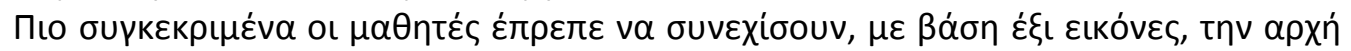

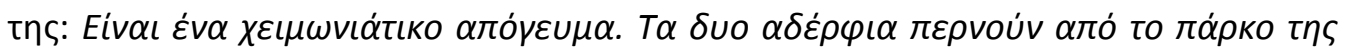

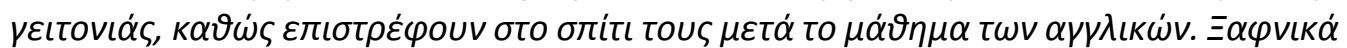

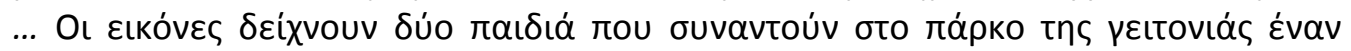

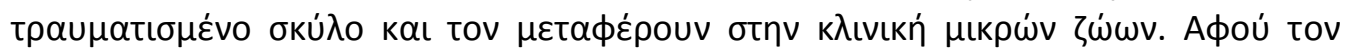

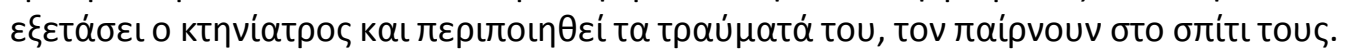

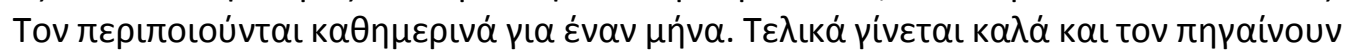

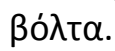

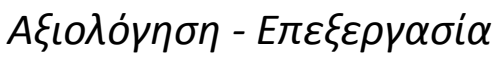

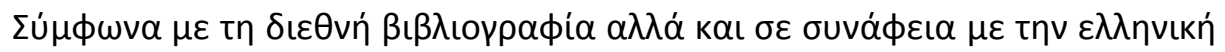

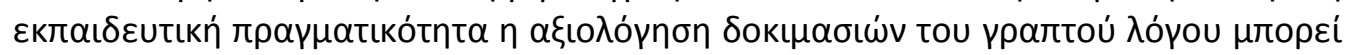

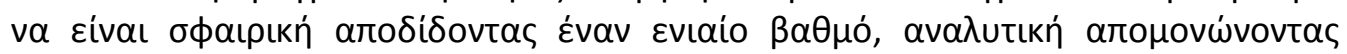

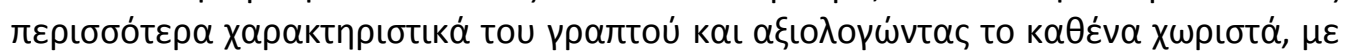

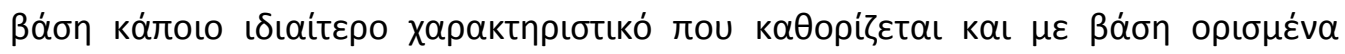

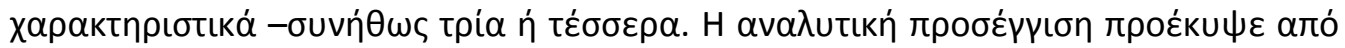

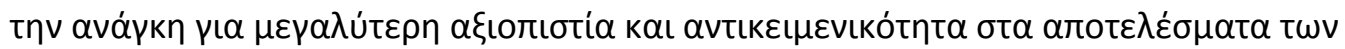

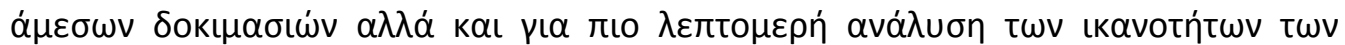

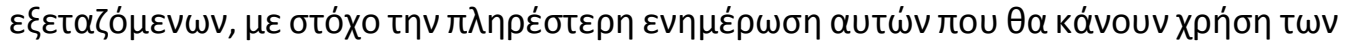


a

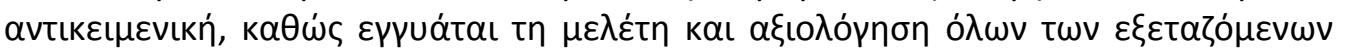

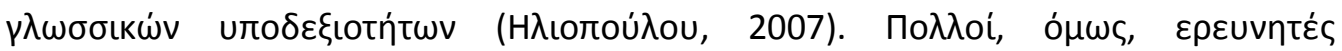

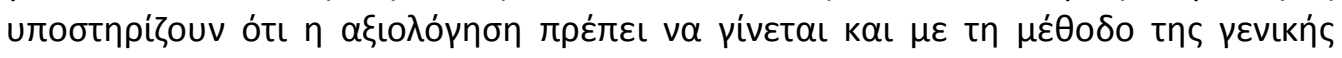

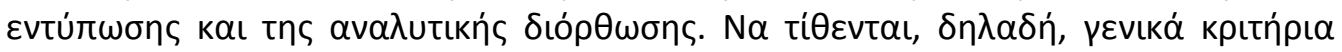

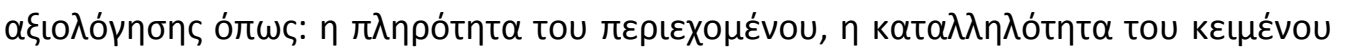

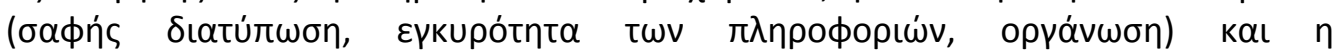

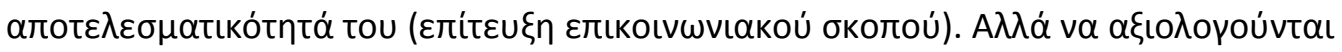

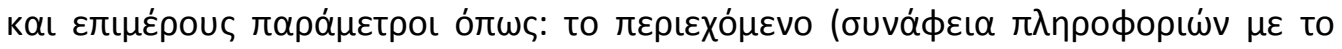

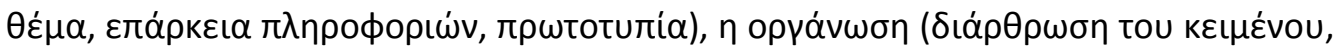

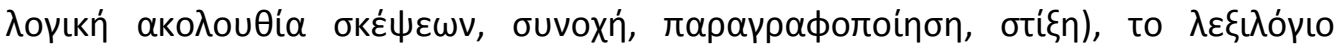

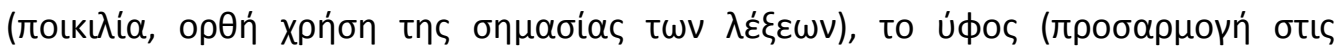

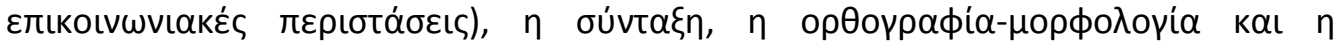

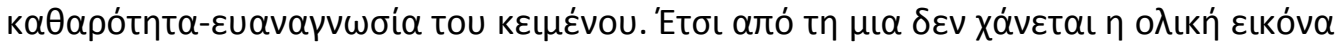

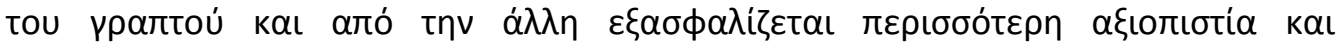

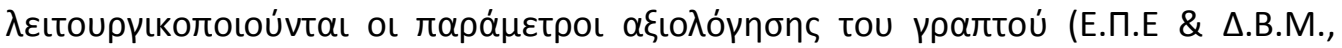
2011).

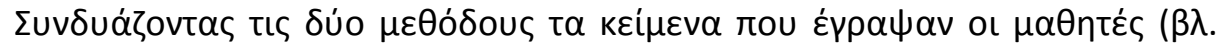

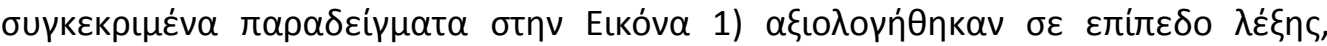

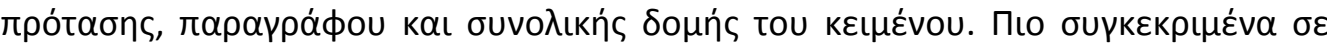

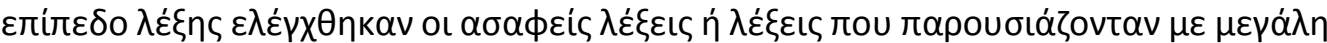

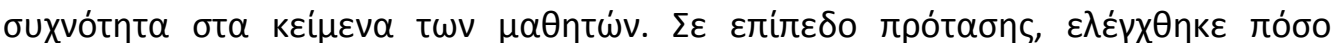

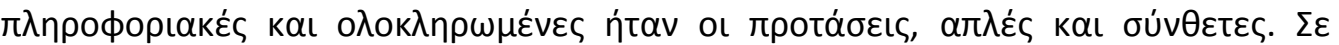

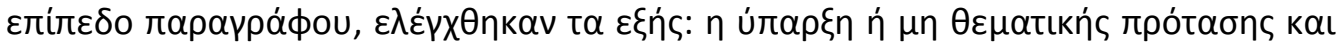

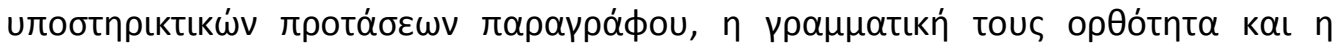

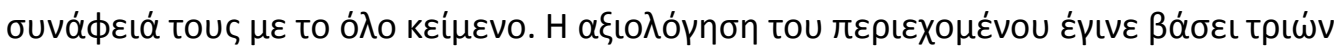

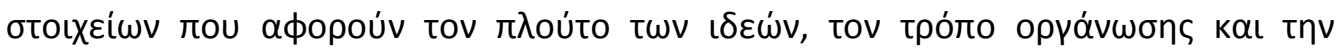

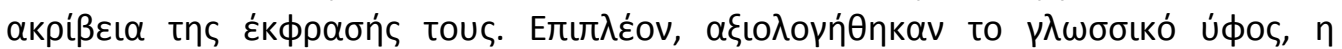

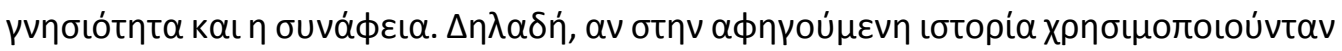

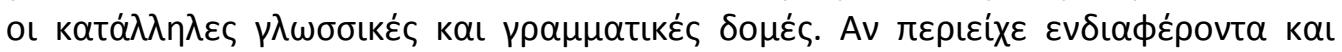

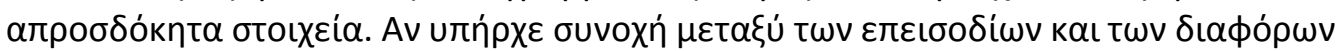

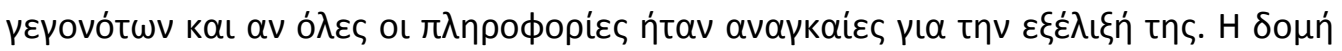

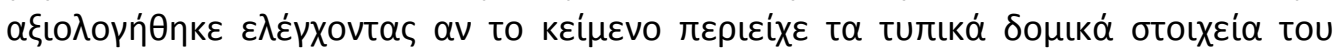

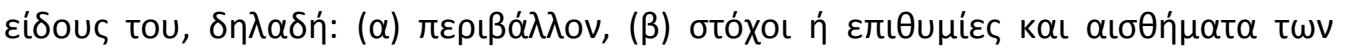

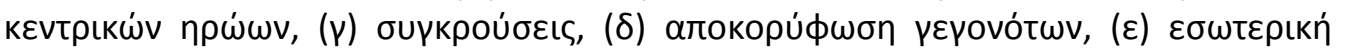

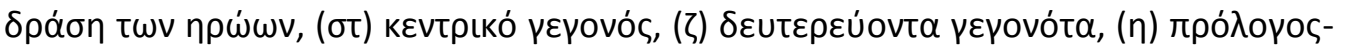

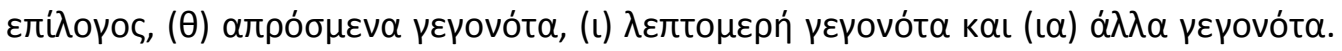

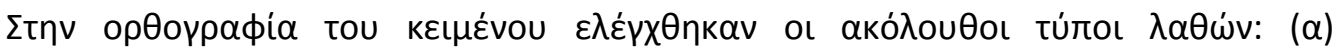

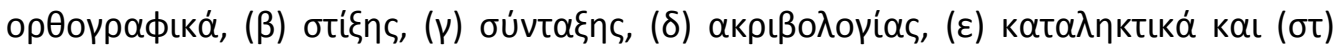
$\theta \varepsilon \mu \alpha \tau$ өка́.

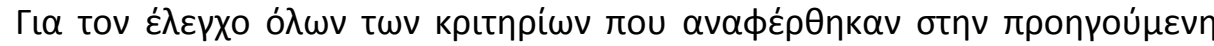

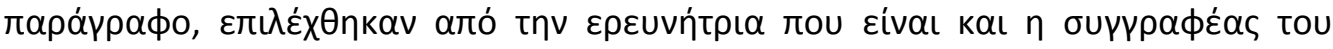

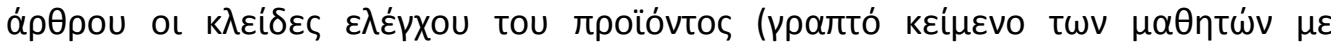

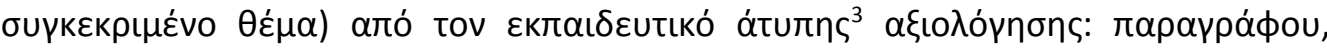

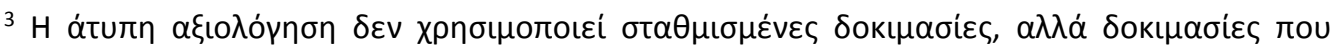

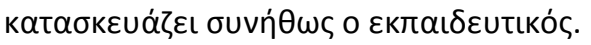




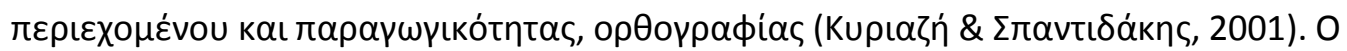

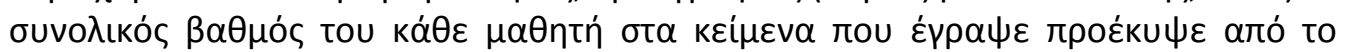

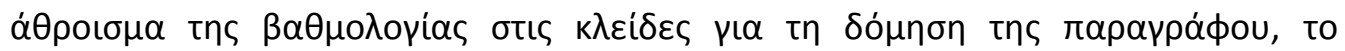

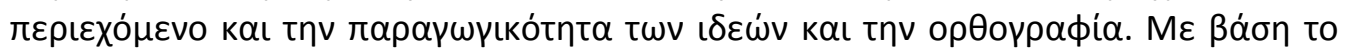

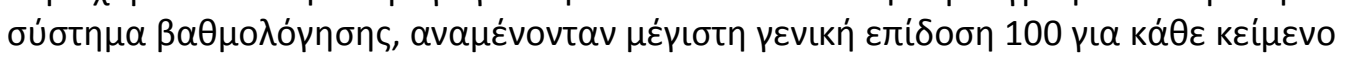

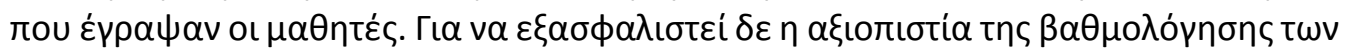

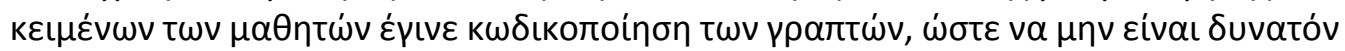

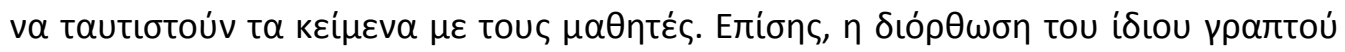

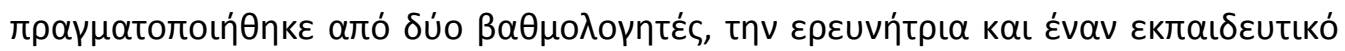

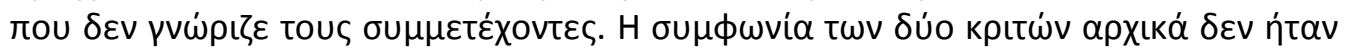

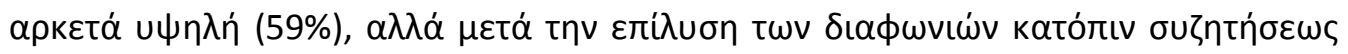

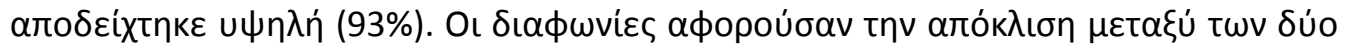

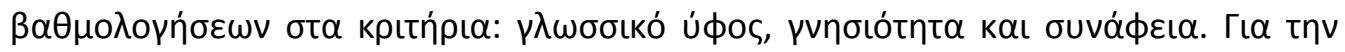

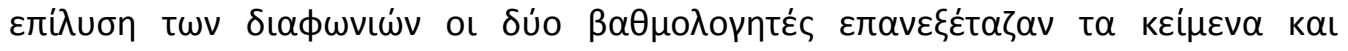

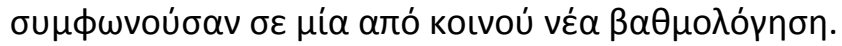




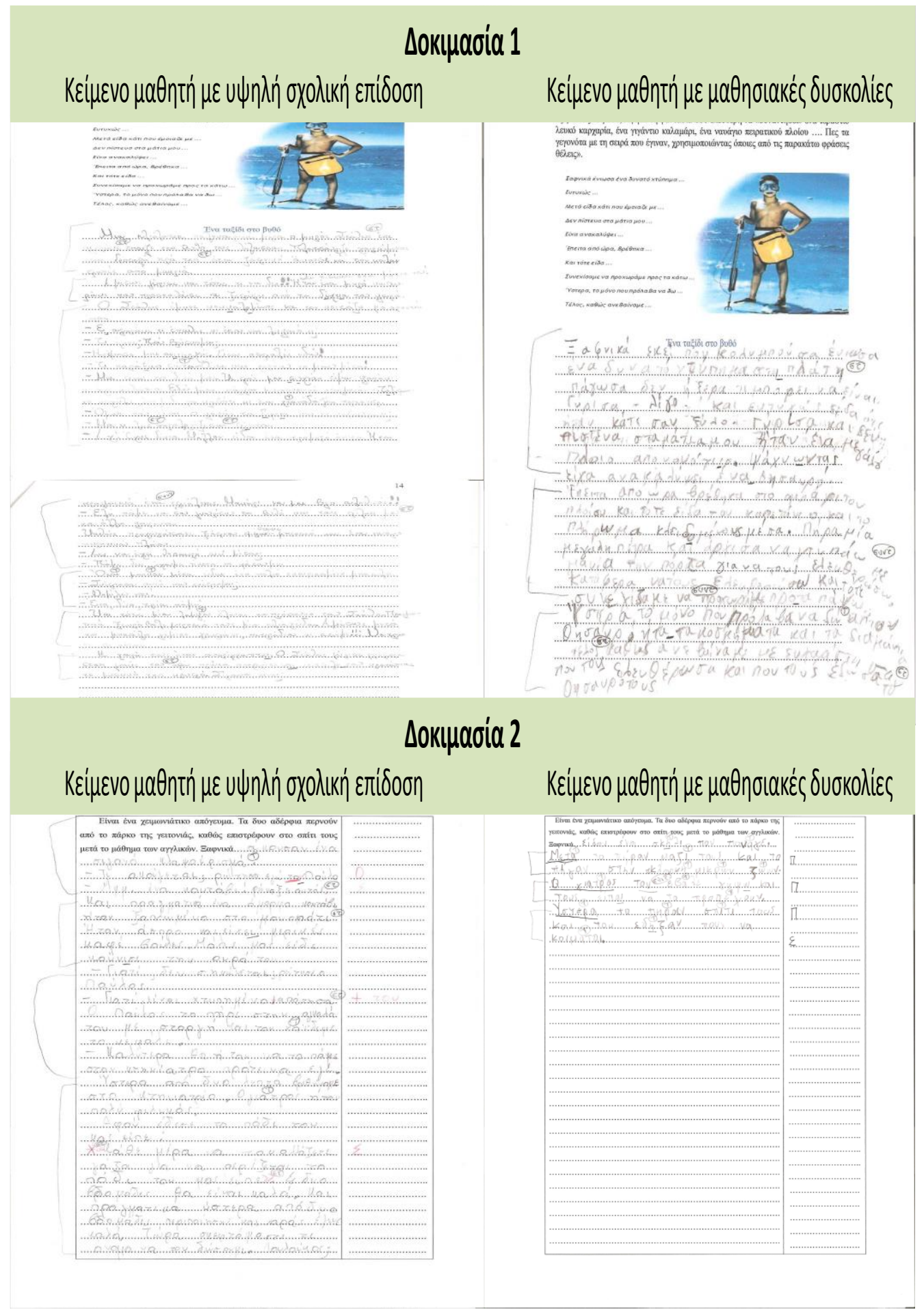

Eıкóva 1.

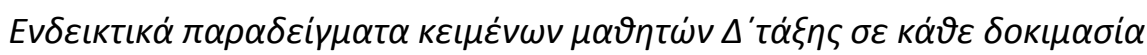




\section{Алотв}

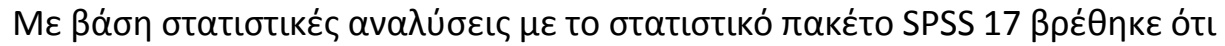

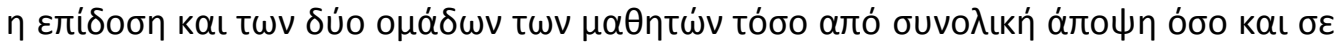

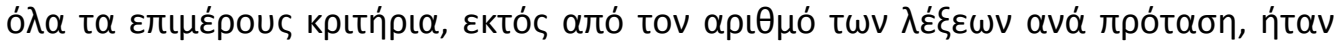

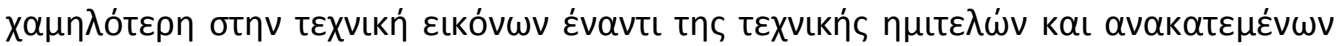

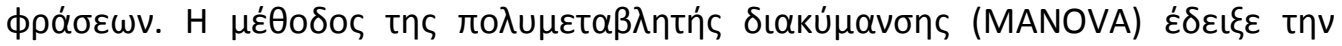

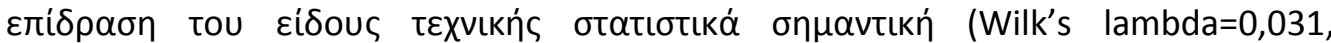

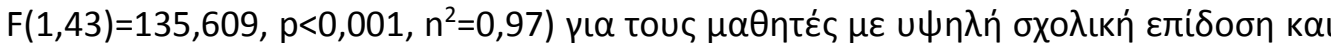

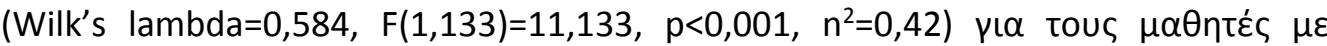

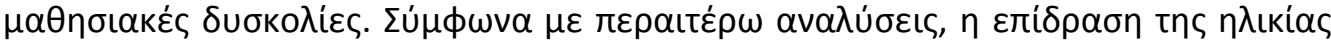

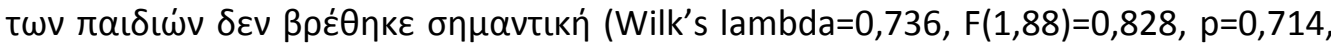

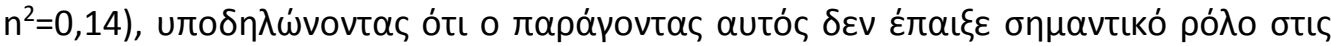

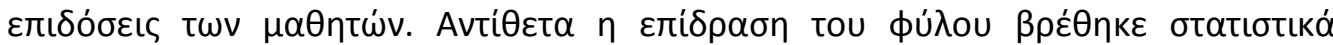

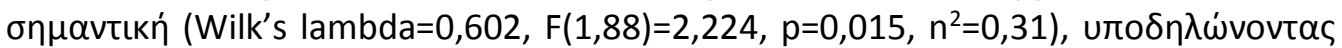

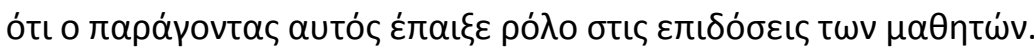

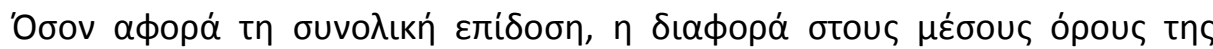

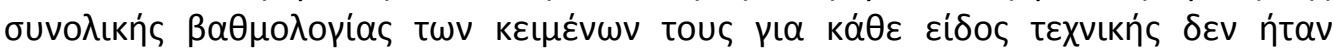

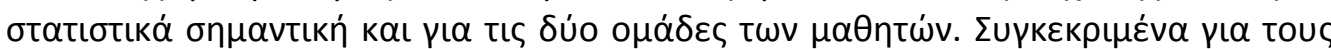

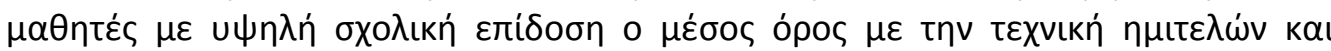

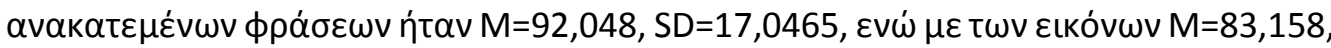

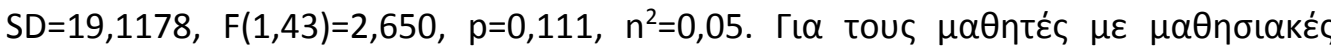

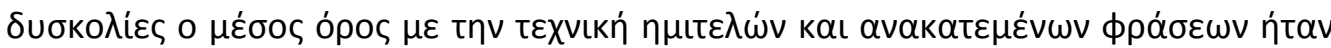

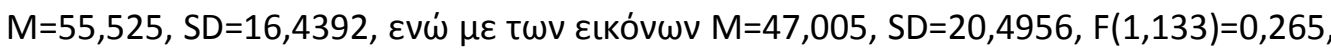

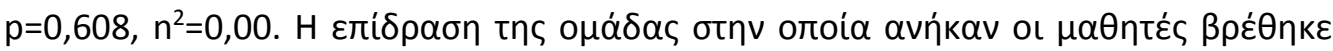

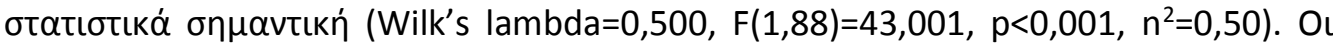

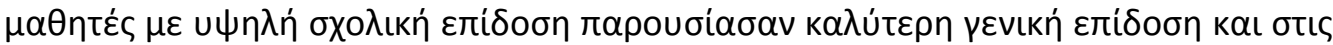

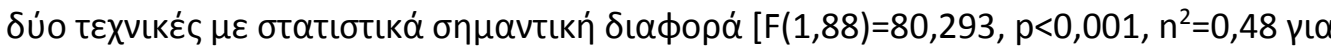

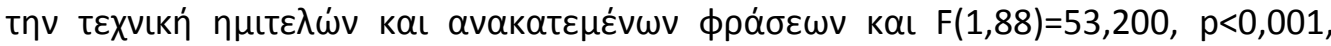

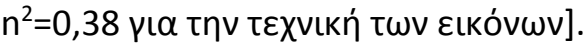

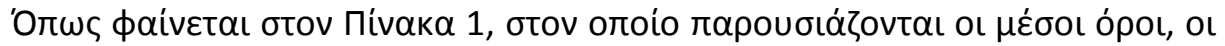

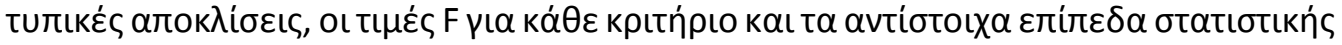

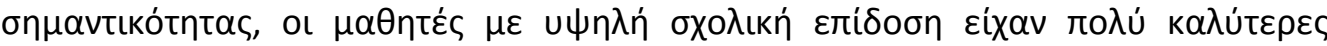

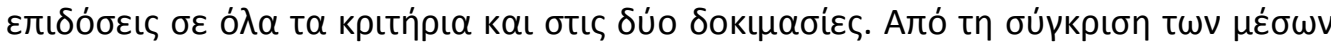

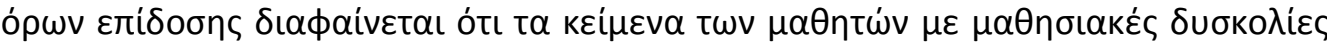

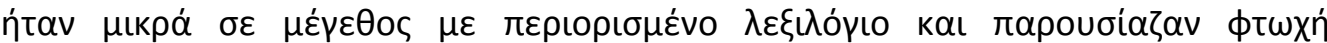

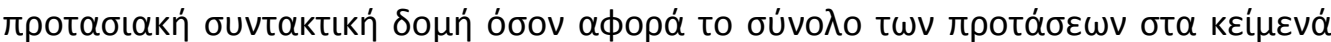

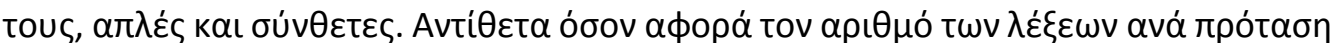

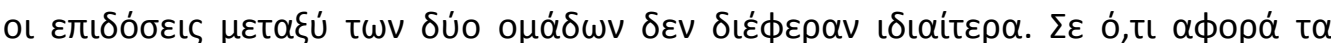

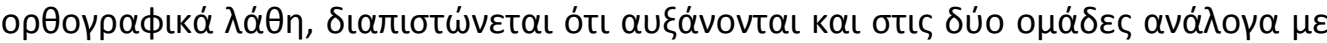

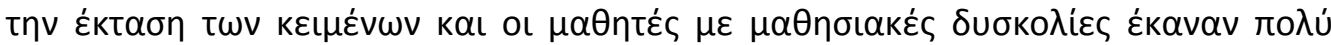

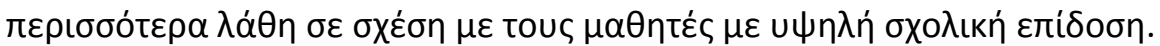




\begin{tabular}{|c|c|c|c|c|c|c|c|}
\hline \multirow[t]{2}{*}{ 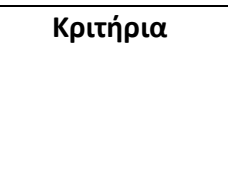 } & \multicolumn{2}{|c|}{ 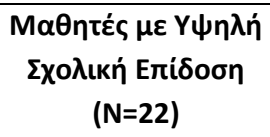 } & \multicolumn{2}{|c|}{ 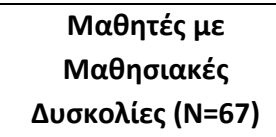 } & \multirow[b]{2}{*}{$F(1,88)$} & \multirow[b]{2}{*}{$\mathbf{p}$} & \multirow[b]{2}{*}{$\mathrm{n}^{2}$} \\
\hline & $\mathbf{M}$ & SD & $M$ & SD & & & \\
\hline $\begin{array}{c}\text { Apı } \theta \mu o ́ \varsigma \wedge \text { } \wedge \dot{\xi} \varepsilon \omega \nu \\
\left(\Delta_{1}\right)\end{array}$ & 174,86 & 52,963 & 92,25 & 35,009 & 70,335 & 0,0001 & 0,45 \\
\hline 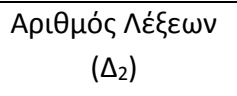 & 102,90 & 42,532 & 59,00 & 24,181 & 36,274 & 0,0001 & 0,29 \\
\hline 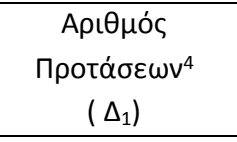 & 18,40 & 6,594 & 10,28 & 3,797 & 51,011 & 0,0001 & 0,37 \\
\hline 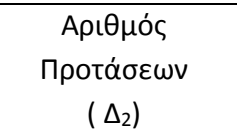 & 9,90 & 5,060 & 6,41 & 2,818 & 16,535 & 0,0001 & 0,16 \\
\hline 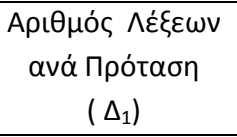 & 9,63 & 1,292 & 9,13 & 2,201 & 1,023 & 0,315 & 0,01 \\
\hline 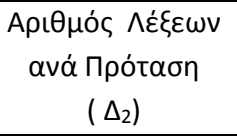 & 11,13 & 2,623 & 9,50 & 2,792 & 1,0253 & 0,018 & 0,06 \\
\hline $\begin{array}{c}\text { Apı } \theta \text { uós } \wedge \alpha \theta \dot{\omega} v \\
\left(\Delta_{1}\right)\end{array}$ & 9,50 & 6,815 & 30,97 & 19,963 & 24,348 & 0,0001 & 0,22 \\
\hline $\begin{array}{c}\text { Apı } \theta \mu o ́ \varsigma, ~ \wedge \alpha \theta \dot{\omega} v \\
\left(\Delta_{2}\right)\end{array}$ & 4,77 & 3,611 & 20,20 & 12,562 & 32,120 & 0,0001 & 0,27 \\
\hline $\begin{array}{c}\Delta o \mu n \\
\left(\Delta_{1}\right)\end{array}$ & 8,84 & 2,702 & 5,11 & 2,016 & 47,591 & 0,0001 & 0,35 \\
\hline $\begin{array}{r}\Delta o \mu n ́ \\
\left(\Delta_{2}\right) \\
\end{array}$ & 5,19 & 2,733 & 3,29 & 1,415 & 17,919 & 0,0001 & 0,17 \\
\hline $\begin{array}{c}\Gamma \lambda \omega \sigma \sigma \text { 'кó úфоৎ } \\
\left(\Delta_{1}\right)\end{array}$ & 9,09 & 0,683 & 7,26 & 1,805 & 21,266 & 0,0001 & 0,20 \\
\hline 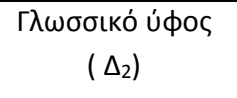 & 9,01 & 0,334 & 6,42 & 2,330 & 26,726 & 0,0001 & 0,24 \\
\hline $\begin{array}{c}\text { Гvทбเótnta } \\
\left(\Delta_{1}\right)\end{array}$ & 9,77 & 0,528 & 8,38 & 1,817 & 12,345 & 0,001 & 0,12 \\
\hline $\begin{array}{c}\text { Гvnбlótnta } \\
\left(\Delta_{2}\right)\end{array}$ & 7,33 & 0,660 & 6,76 & 2,365 & 24,850 & 0,0001 & 0,22 \\
\hline 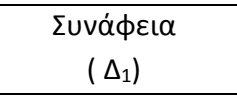 & 9,81 & 0,501 & 8,10 & 1,923 & 16,957 & 0,0001 & 0,16 \\
\hline 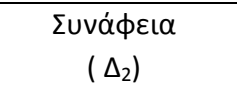 & 9,27 & 0,810 & 6,64 & 2,234 & 29,080 & 0,0001 & 0,25 \\
\hline
\end{tabular}

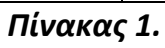

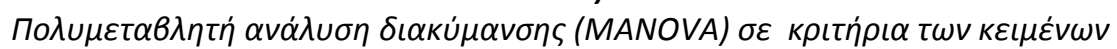

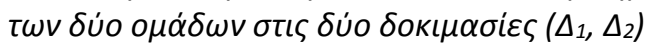

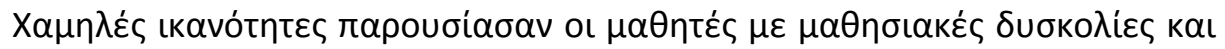

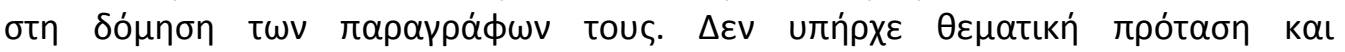

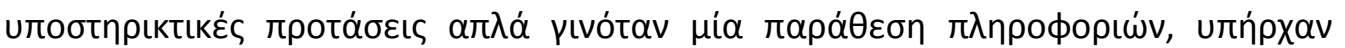




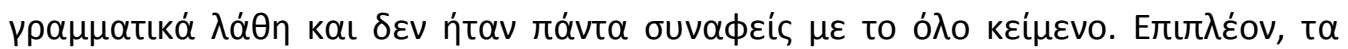

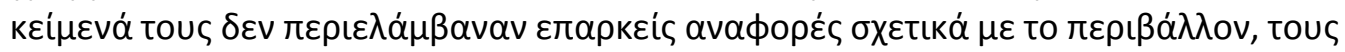

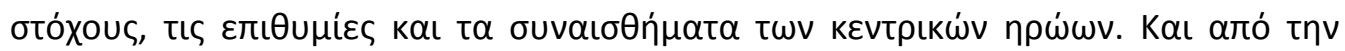

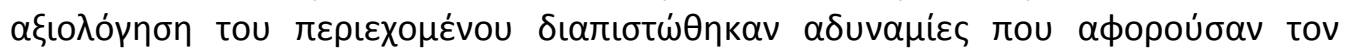

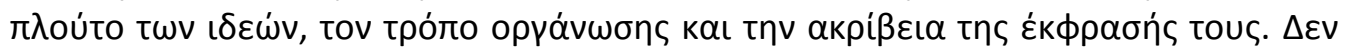

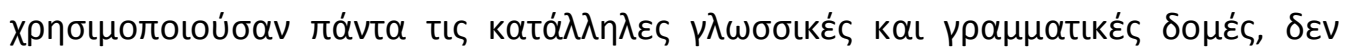

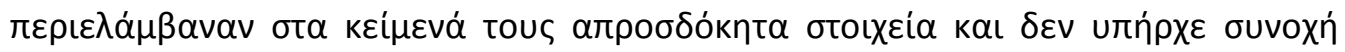

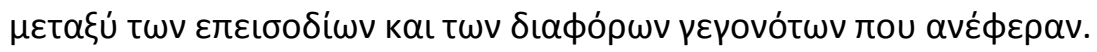

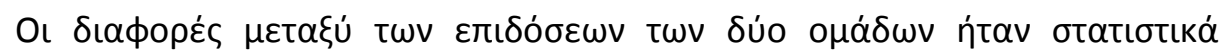

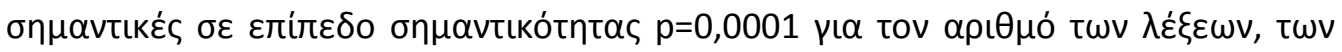

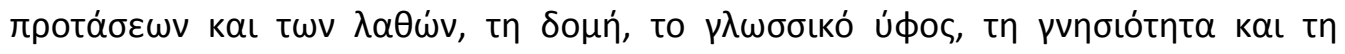

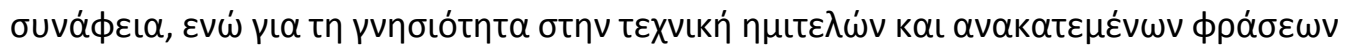

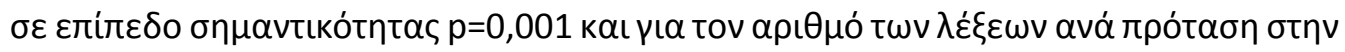

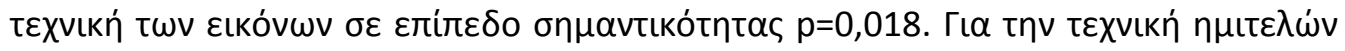

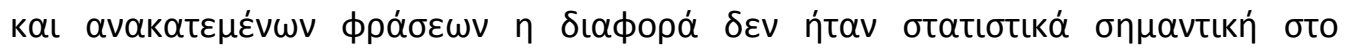

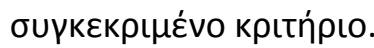

\begin{tabular}{|c|c|c|c|c|c|c|c|c|}
\hline \multicolumn{2}{|c|}{ 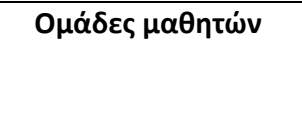 } & \multicolumn{2}{|c|}{ 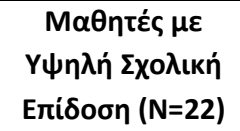 } & \multicolumn{2}{|c|}{ 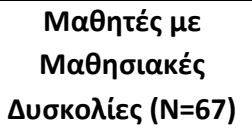 } & \multirow{2}{*}{$\begin{array}{c}\Delta ı \alpha \phi \circ \\
\rho \alpha ́ \\
\varepsilon \pi i ́ \delta \circ \\
\text { ons } \\
\end{array}$} & \multirow[b]{2}{*}{$\mathbf{t}$} & \multirow[b]{2}{*}{ p } \\
\hline 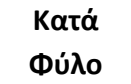 & Avód Tó $\xi \eta$ & $M$ & SD & M & SD & & & \\
\hline & 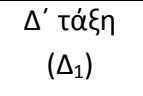 & 91,49 & 26,957 & 59,27 & 9,858 & 32,22 & 4,482 & 0,0001 \\
\hline & 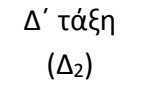 & 90,91 & 20,489 & 47,43 & 21,175 & 43,48 & 4,130 & 0,0001 \\
\hline & $\begin{array}{c}E^{\prime} \tau \alpha \dot{\xi} \eta \\
\left(\Delta_{1}\right)\end{array}$ & 95,98 & 11,249 & 53,34 & 19,053 & 42,64 & 6,382 & 0,0001 \\
\hline & 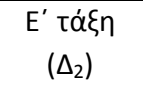 & 83,29 & 21,732 & 48,86 & 17,920 & 34,43 & 4,884 & 0,0001 \\
\hline & $\begin{array}{c}\Sigma T^{\prime} \tau \alpha \dot{\alpha} \eta \\
\left(\Delta_{1}\right)\end{array}$ & 87,96 & 16,512 & 55,30 & 17,391 & 32,65 & 4,312 & 0,0001 \\
\hline & $\begin{array}{c}\Sigma T^{\prime} \tau \dot{\alpha} \xi \eta \\
\left(\Delta_{2}\right)\end{array}$ & 78,15 & 15,656 & 42,01 & 25,512 & 36,14 & 3,615 & 0,002 \\
\hline $\begin{array}{c}\text { Корітбıа } \\
\left(\Delta_{1}\right)\end{array}$ & & 96,58 & 16,920 & 55,45 & 14,506 & 41,13 & 8,286 & 0,0001 \\
\hline 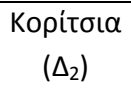 & & 91,51 & 182,084 & 45,96 & 21,220 & 45,55 & 6,956 & 0,0001 \\
\hline $\begin{array}{c}\text { Ayópıa } \\
\left(\Delta_{1}\right)\end{array}$ & & 84,10 & 15,059 & 55,63 & 17,805 & 28,47 & 4,221 & 0,0001 \\
\hline $\begin{array}{c}\text { Apópıa } \\
\left(\Delta_{2}\right)\end{array}$ & & 68,52 & 10,232 & 47,89 & 20,215 & 20,63 & 2,799 & 0,007 \\
\hline
\end{tabular}

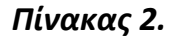

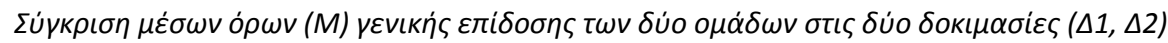

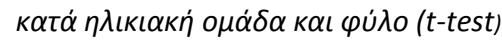




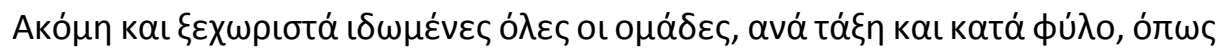

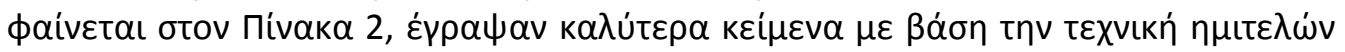

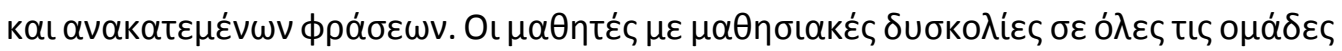

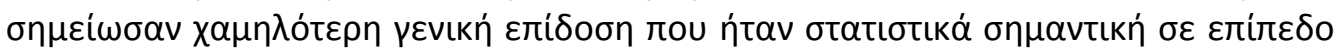

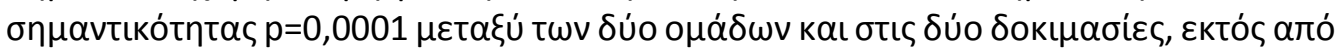

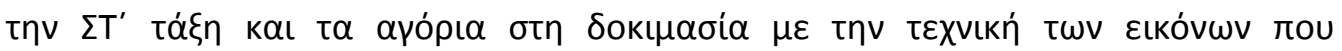

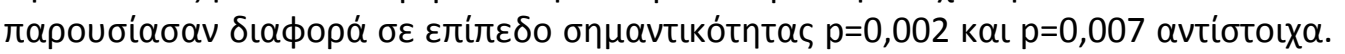

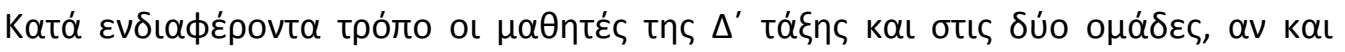

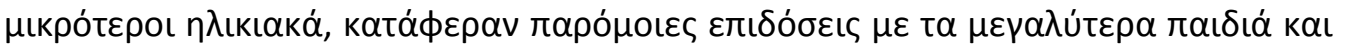

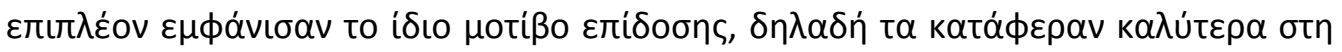

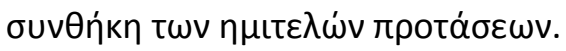

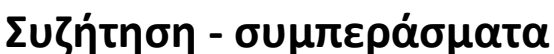

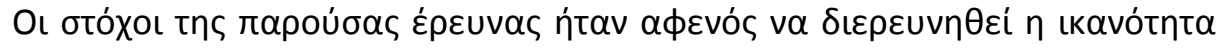

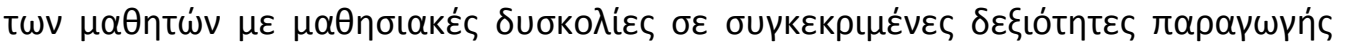

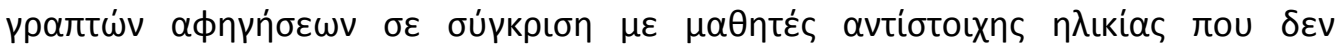

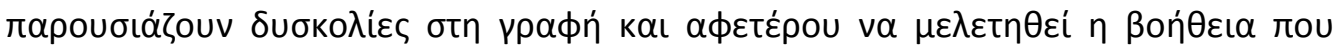

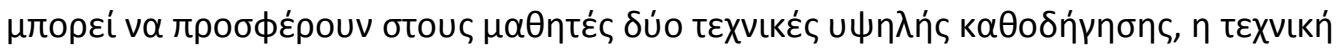

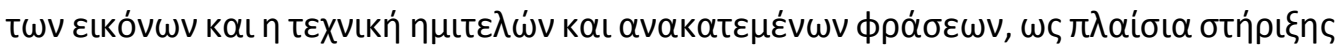

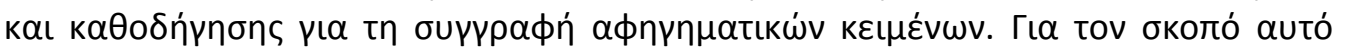

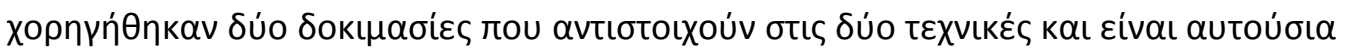

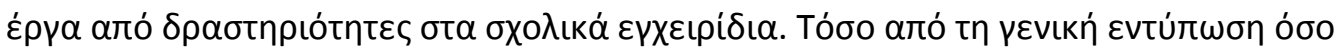

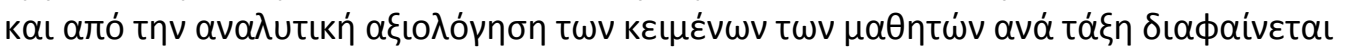

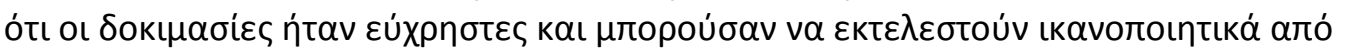

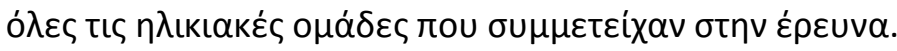

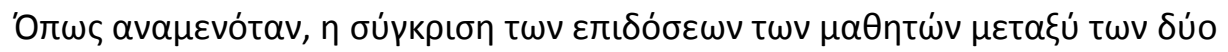

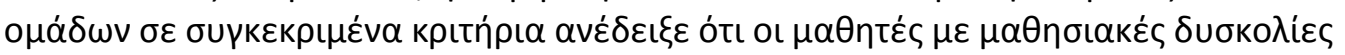

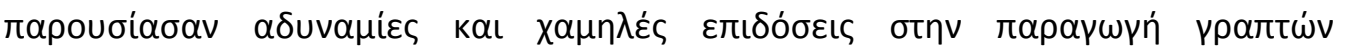

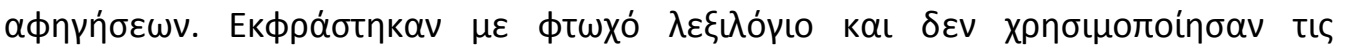

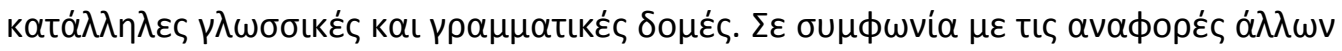

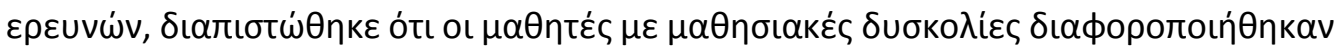

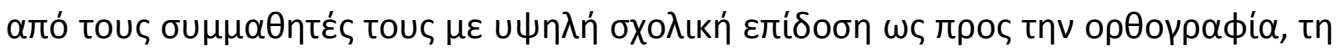

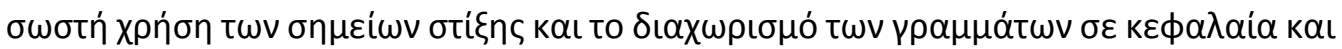

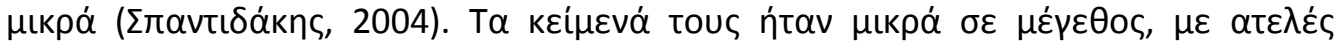

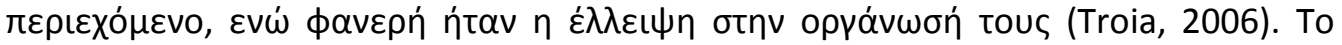

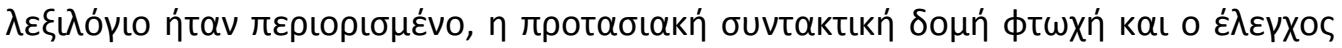

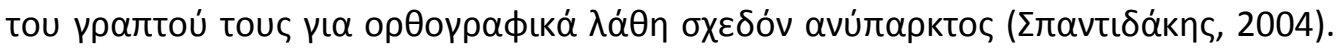

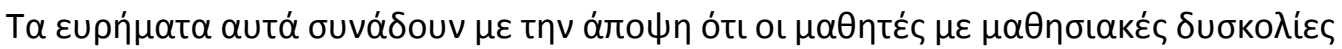

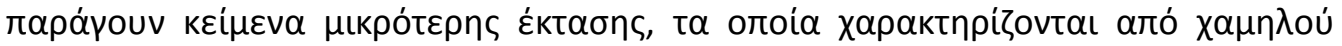

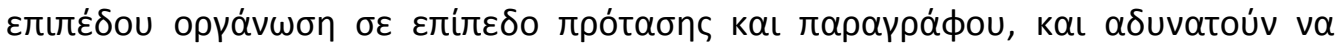

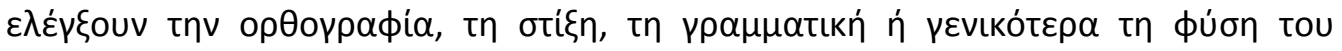

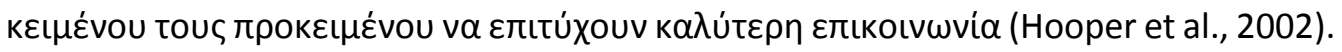

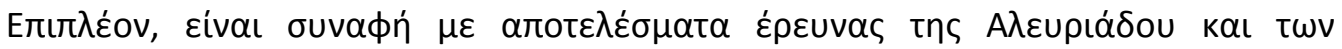

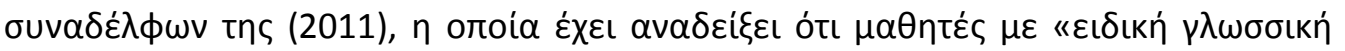

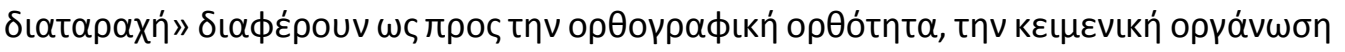

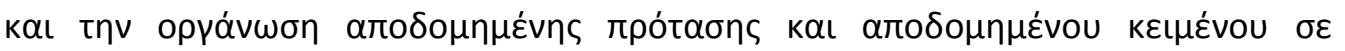




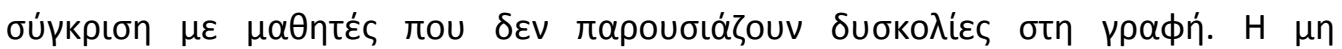

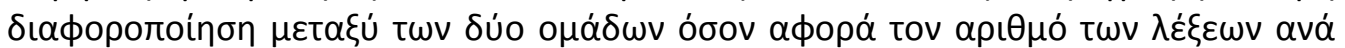

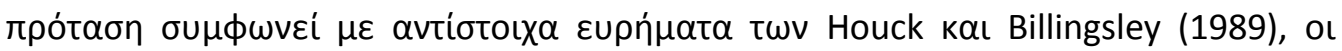

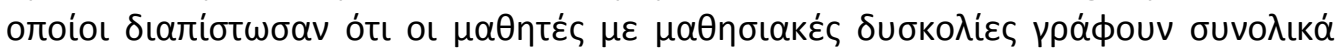

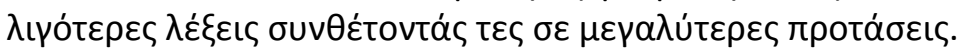

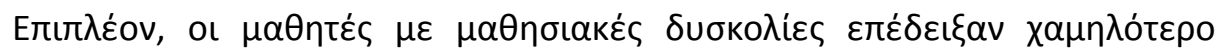

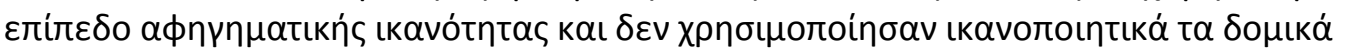

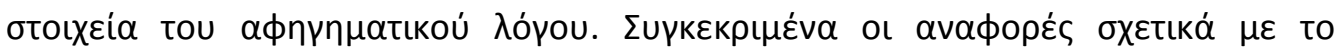

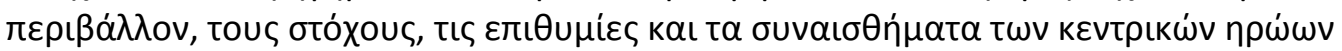

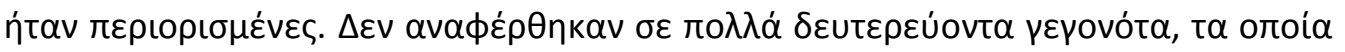

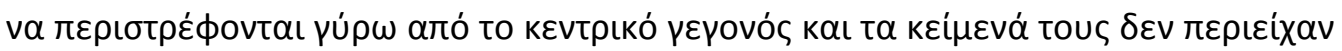

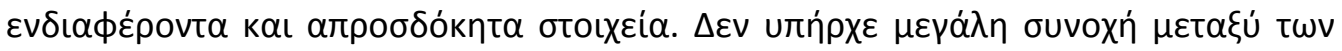

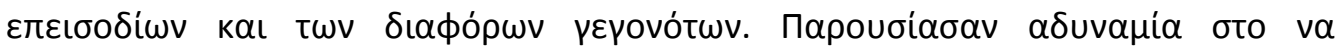

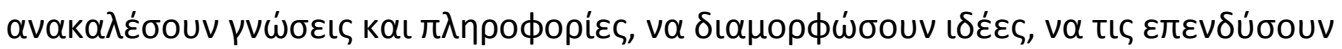

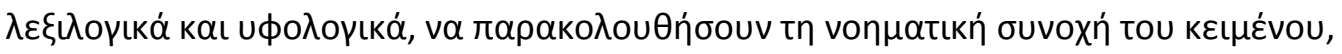

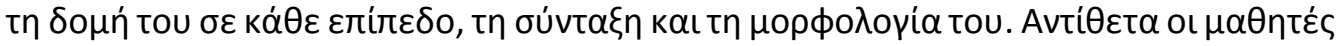

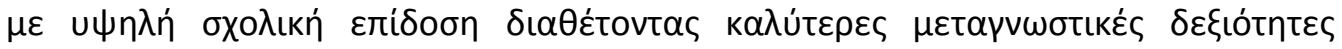

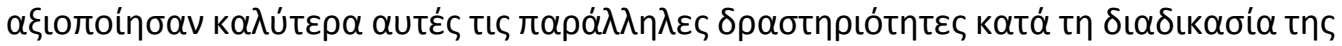

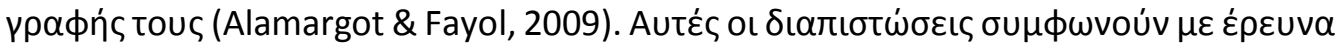

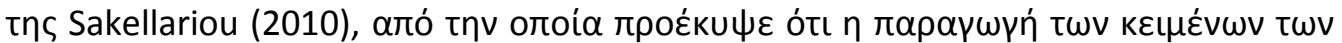

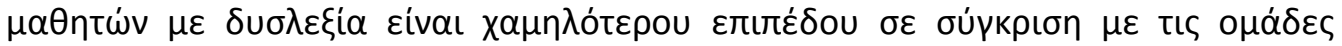

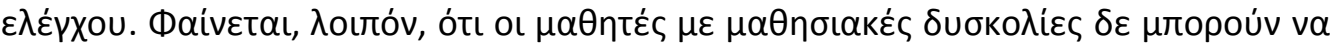

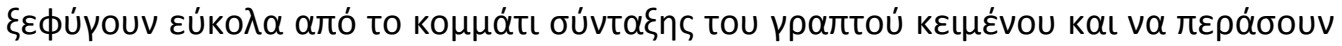
бто бuүүрафıко́ (Newcomer \& Barenbaum, 1991).

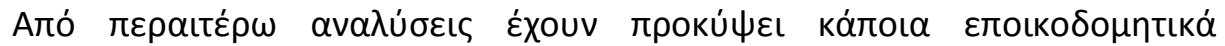

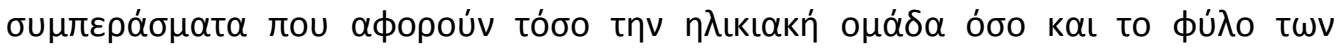

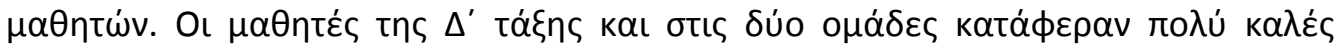

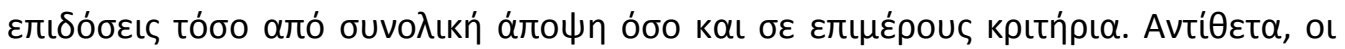

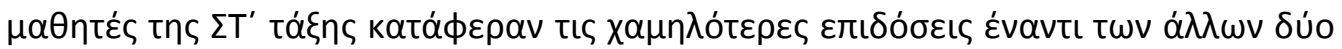

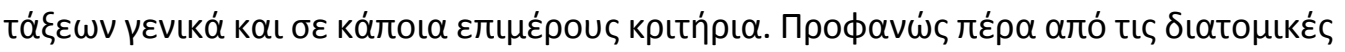

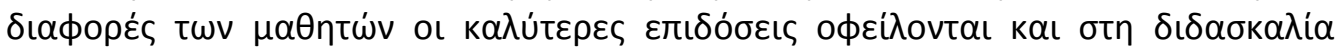

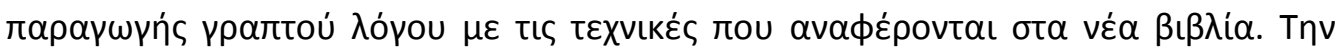

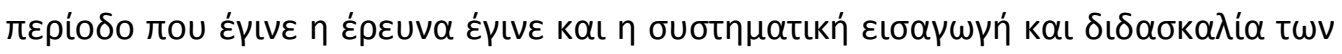

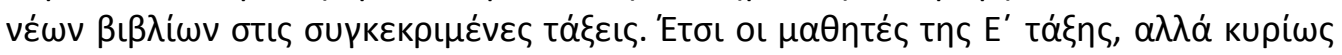

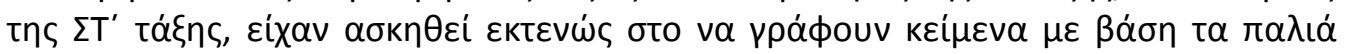

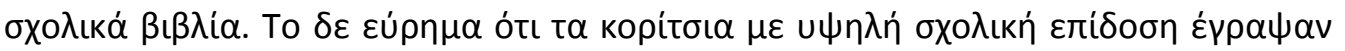

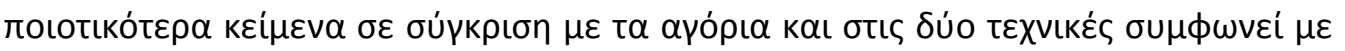

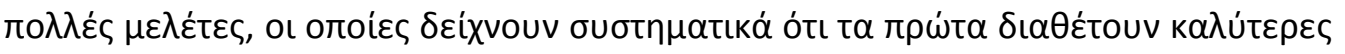

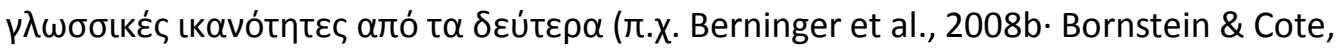

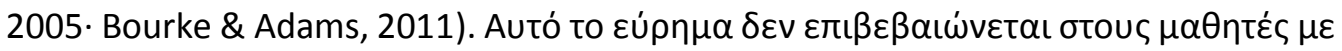

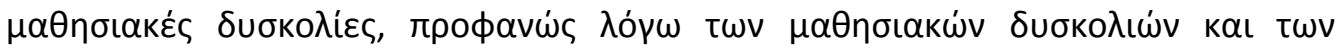

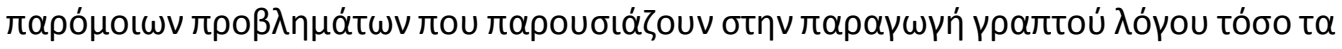

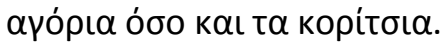

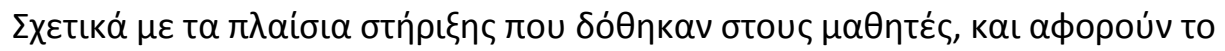

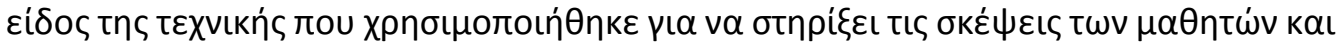

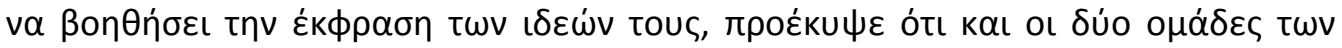




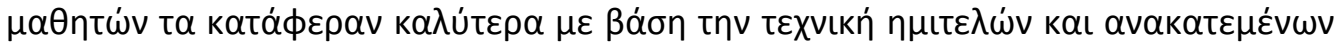

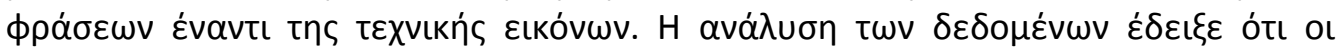

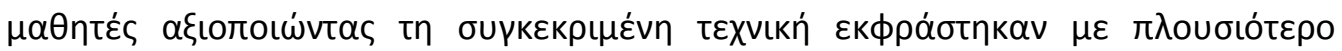

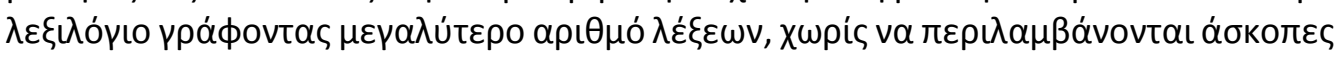

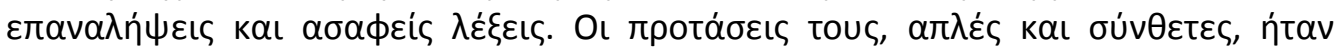

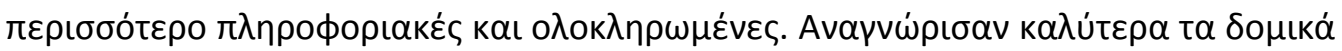

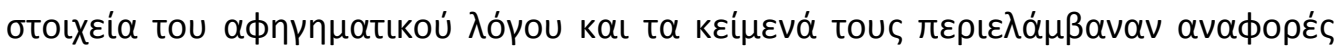

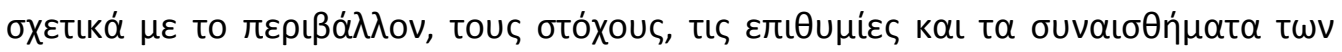

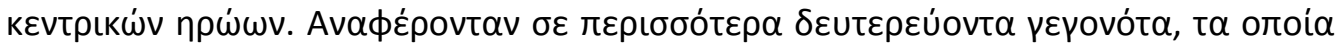

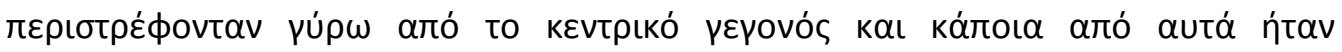

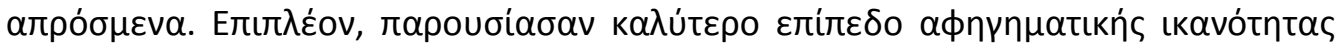

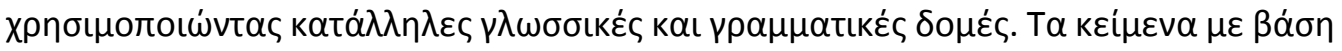

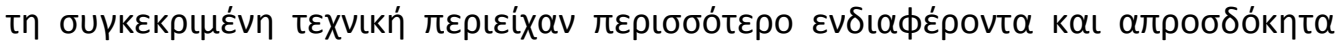

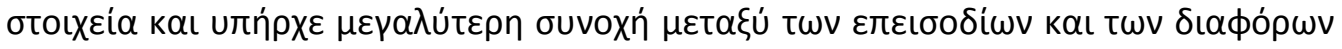

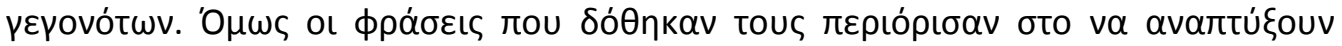

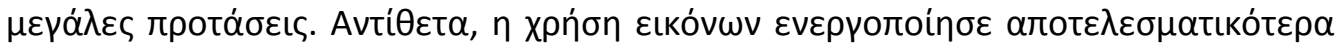

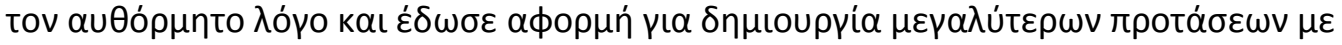

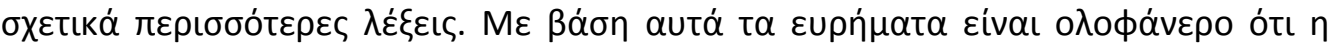

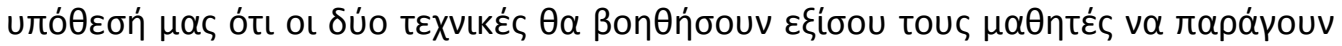

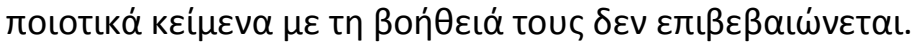

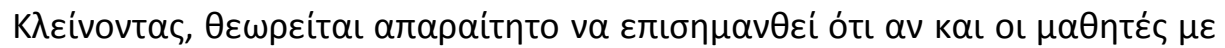
$\mu \alpha \theta \eta \sigma \iota \alpha \kappa \varepsilon$ ऽ $\delta$

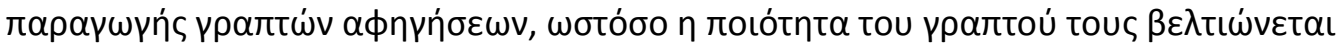

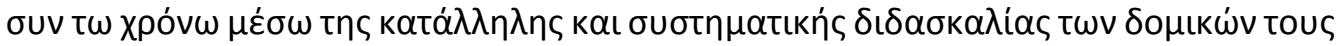

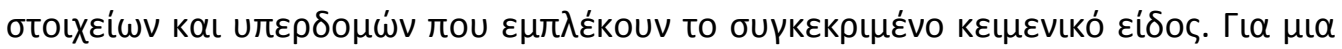

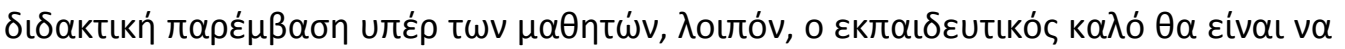

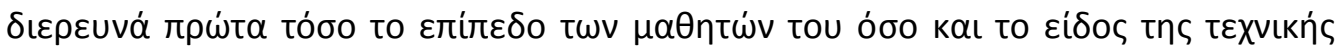

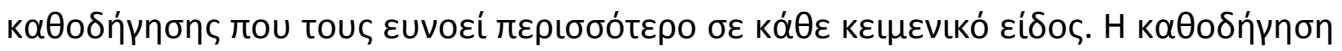

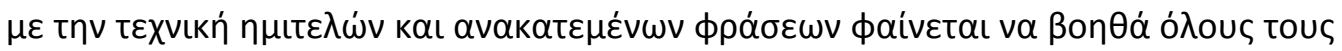

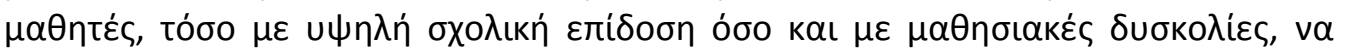

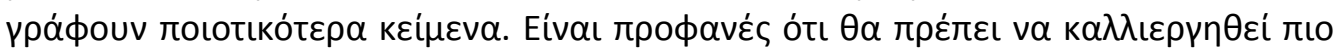

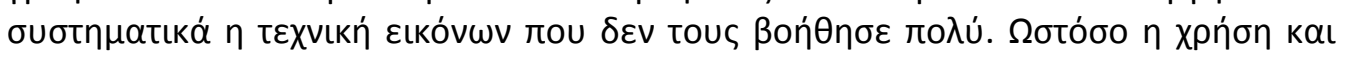

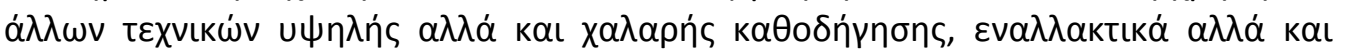

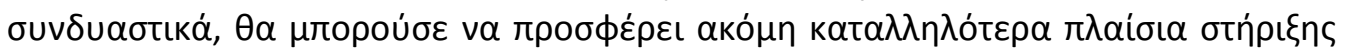

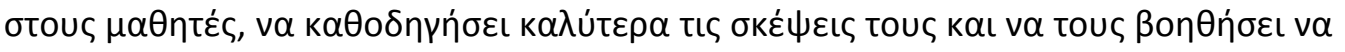

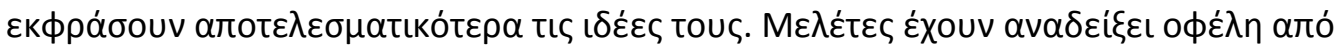

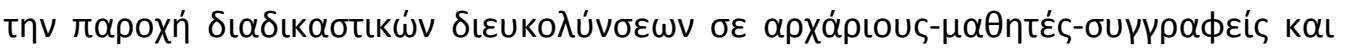

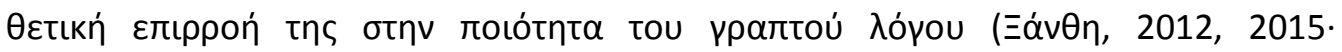

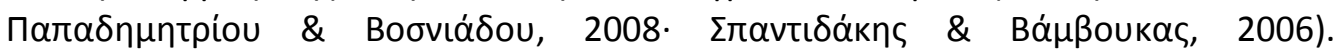

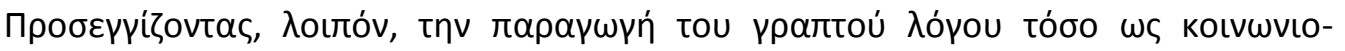

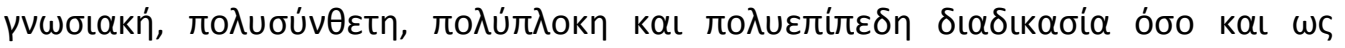

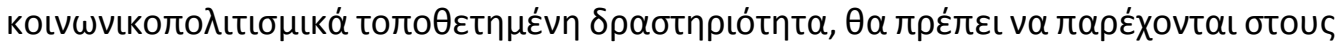

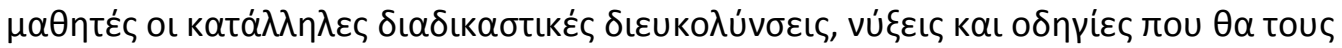

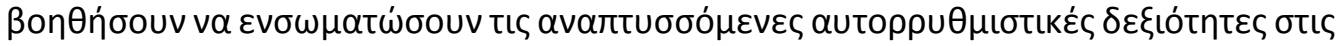

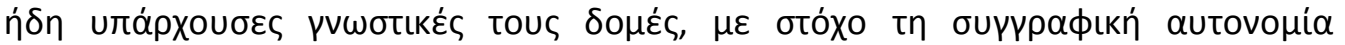

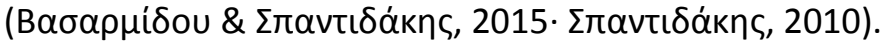




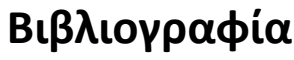

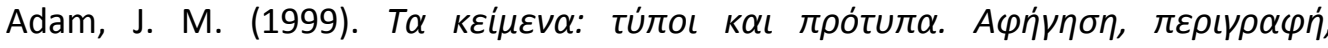

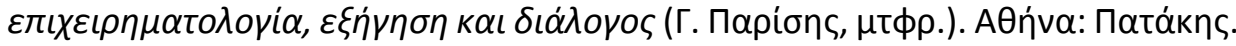

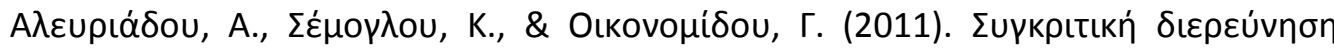

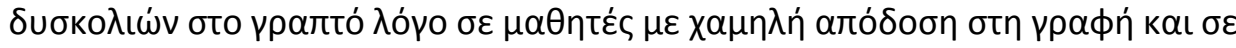

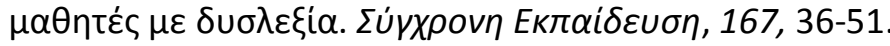

Almargot, D., \& Fayol, M. (2009). Modeling the development of written composition. In R. Beard, D., Myhill, J. Riley, \& M. Nystrand (Eds.), The Sage handbook of writing development (pp.23-47). London, UK: Sage.

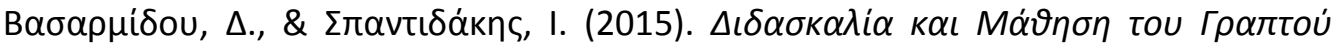

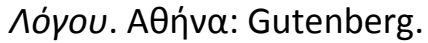

Berninger, V., Nielsen, K., Abbott, R., Wijsman, E., \& Raskind, W. (2008b). Gender differences in severity of writing and reading disabilities. Journal of School Psychology, 46, 151-172.

Berninger, V. W., Mizokowa, D. T., \& Bragg, R. (1991). Theory-based diagnosis and remediation of writing disabilities. Journal of School Psychology, 29, 57-79.

Bonheim, H. (1992). The narrative modes: techniques of the short story. Cambridge U.K.: D.S. Brewer.

Bornstein, M. H., \& Cote, L. R. (2005). Expressive vocabulary in language learners from two ecological settings in three language communities. Infancy, 7, 299-316.

Bourke, L., \& Adams, A. (2011). Is it difference in language skills and working memory that account for girls being better at writing than boys. Journal of Writing Research, 3(3), 249-277.

Bruner, J. (1978). The role of dialogue in language acquisition. In A. Sinclair, R. J. Jarvella, \& J. M. Levert (Eds.), The Child's conception of language acquisition (pp. 241-256). New York, NY: Springer-Verlag.

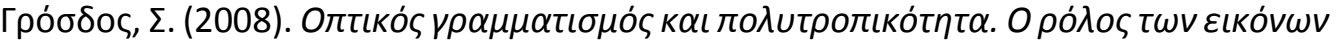

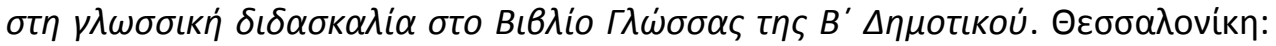

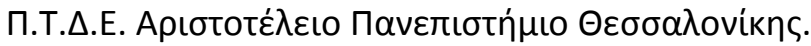

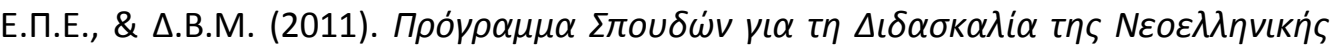

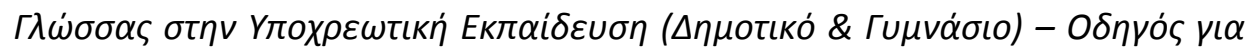

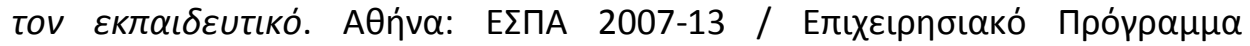

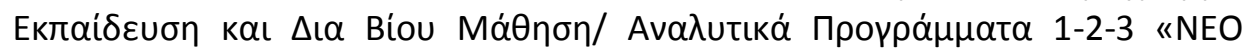

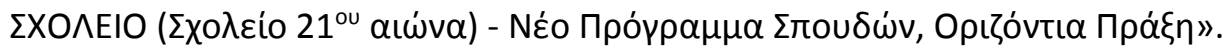

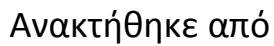
http://www.moec.gov.cy/dde/diapolitismiki/ilektroniko yliko/odigos glossas gia dimotiko gymnasio.pdf

Fitzgerald, J. (1989). Research on stories. In K. D. Mut (Ed.), Children's Comprehension of Text (pp. 2-36). Newark. DE: International Reading Association.

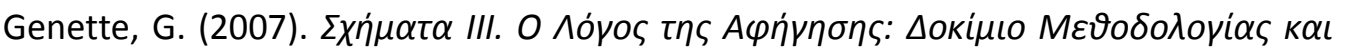

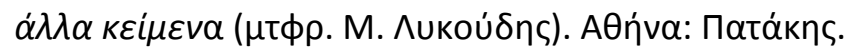




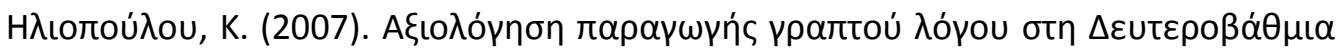

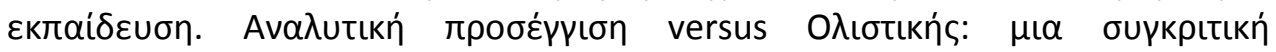

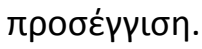

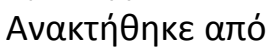
http://ipeir.pde.sch.gr/educonf/2/06DefterovathmiaEkpedefsi/iliopoulou/iliopou lou.pdf

Hooper, S. R. (2002). The Language of Written Language: An Introduction to the Special Issue. Journal of Learning Disabilities, 35 (1), 2-6.

Houck, C. K., \& Billingsley, B. S. (1989). Written expression of students with and without learning disabilities: Differences across grades. Journal of Learning Disabilities, 22, 561-572.

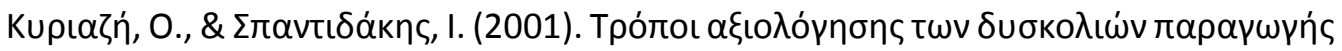

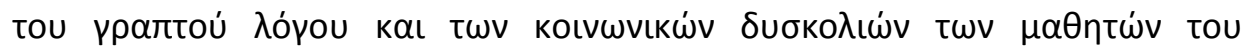

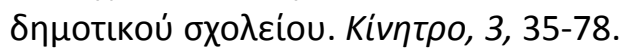

Magos, K., \& Kontogianni, A. (2009). «When Ali meets Angeliki»: A Research of Student Teachers Perceptions for the Ethnic Other. In the Proceedings of the International Congress of Comparative Literature and the Teaching of Literature and Language We Speak the Same Culture (pp. 475-489). Ankara, Turkey: Gazi University.

Mandler, J. M. (1984). Stories, script and scenes: Aspects of schema theory. Hillsdale, NJ: Lawrence Erlbaum Associates.

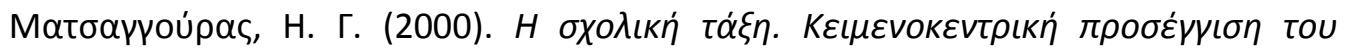

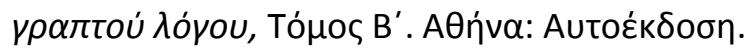

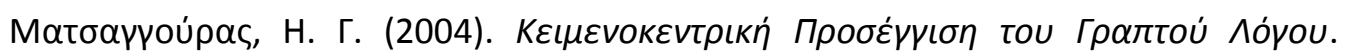

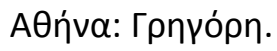

Newcomer, P. L., \& Barenbaum, E. M. (1991). The written composing ability of children with learning disabilities. A review of literature from 1980 to 1990. Journal of Learning Disabilities, 24, 578-593.

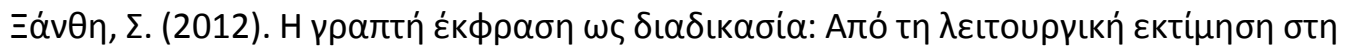

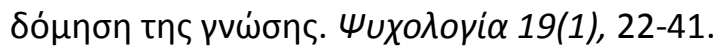

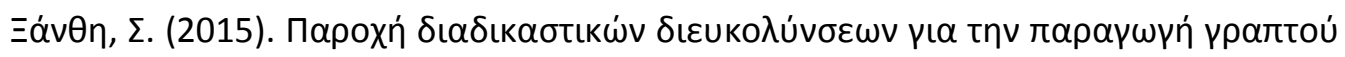

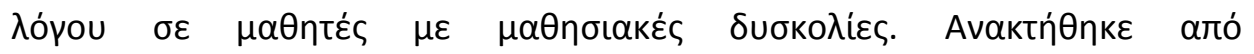
http://www.specialeducation.gr/frontend/article.php?aid=764\&cid=74

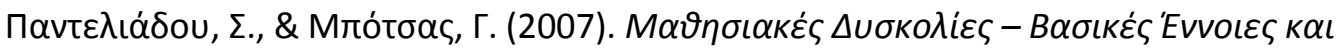

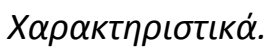

АvактиंӨпкє ато́

http://www.specialeducation.gr/files4users/files/pdf/teyxos a.pdf

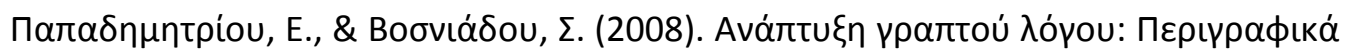

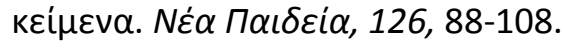

Quinlan, T. (2004). Speech recognition technology and students with writing difficulties: Improving fluency. Journal of Educational Psychology, 96(2), 337-346. 
Sakellariou, E. (2010). Does memory play a part in the quality of the written product? The impact of the working memory system and other cognitive processes in writing performance in students with dyslexia (Unpublished doctoral dissertation). University of Southampton, UK.

Scardamalia, M., \& Bereiter, C. (1987). Knowledge telling and knowledge transforming in written composition. In S. Rosenberg (Ed.), Advances in Applied. Psycholinguistics: Vol. 2: Reading, Writing, and Language Learning (pp. 142-175). Cambridge, UK: Cambridge University Press.

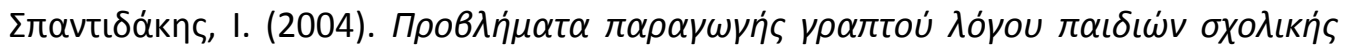

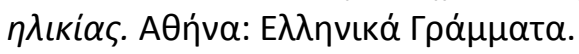

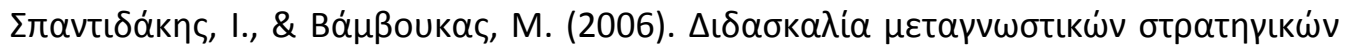

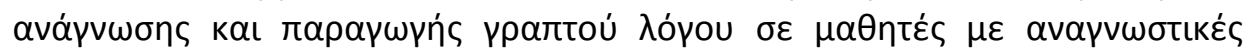

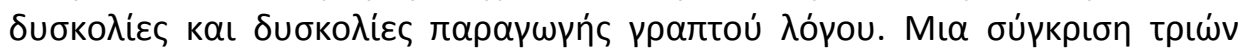

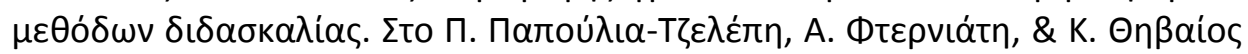

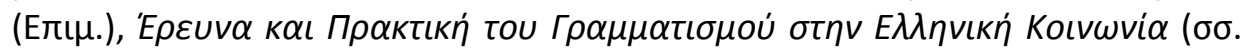

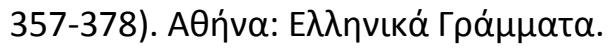

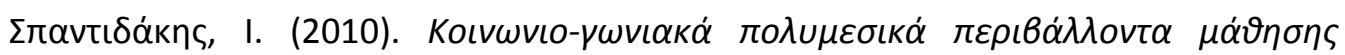

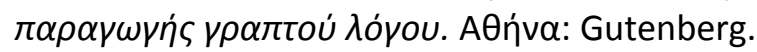

Torrance, M., \& Galbraith, D. (2006). The processing demands of writing. In C. A. MacArthur, S. Graham, \& J. Fitzgerald (Eds.), Handbook of writing research. New York, NY: Guilford Publications.

Troia, G. A. (2006). Writing instruction for students with learning disabilities. In C. A. MacArthur, S. Graham, \& J. Fitzgerald (Eds.), Handbook of writing research (pp. 324-336). New York, NY: The Guilford Press.

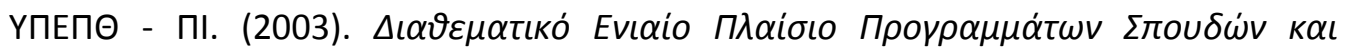

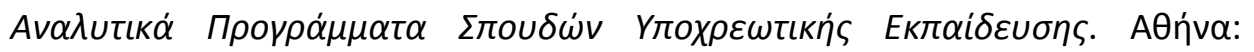

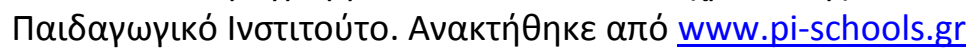

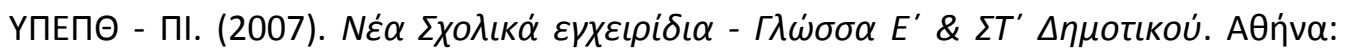

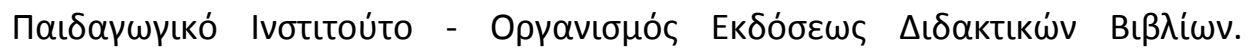

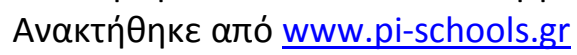

